\title{
14. GEOCHEMISTRY OF SEDIMENTS IN THE WESTERN CENTRAL ATLANTIC, DSDP LEG 39
}

E.M. Emelyanov, P.P. Shirshov Institute of Oceanology, USSR Academy of Sciences, Kaliningrad, USSR

\section{INTRODUCTION}

Twenty chemical elements were studied and $\mathrm{CaCO}_{3}$, $\mathrm{CO}_{2}$, and $\mathrm{C}$ (organic) were determined by the modified volumetric chemical method (Sokolov and Sokolova, 1975). Other samples were decomposed to determine $\mathrm{Fe}$, $\mathrm{Mn}, \mathrm{Ti}, \mathrm{Na}, \mathrm{O}, \mathrm{K}_{2} \mathrm{O}, \mathrm{Zn}, \mathrm{Cu}, \mathrm{Ni}, \mathrm{Co}, \mathrm{Cr}$, and $\mathrm{Cd}$. Decomposition of the sample $(0.25 \mathrm{gr})$ was accomplished with acid (with the application of $\mathrm{HCl}, \mathrm{HNO}_{3}$, and $\mathrm{HF}$ ) in platinum crucibles. The concentrations of $\mathrm{Na}_{2} \mathrm{O}$ and $\mathrm{K}_{2} \mathrm{O}$ were measured by flame photometry (M-3, FRG), Ti was measured by the photoelectrocolorimeter (FEK-56, U.S.S.R.), and all other elements were analyzed using the atomic-absorption spectrophotometer "Saturn" (U.S.S.R.). Phosphorus was analyzed in a separate sample ( $0.5 \mathrm{gr})$, decomposed in aqua regia, i.e. a mixture of $\mathrm{HCl}$ ( 3 parts) and $\mathrm{HNO}_{3}(1$ part). The concentration of $\mathrm{P}$ was determined photocolorimetrically by FEK -56 . Ba, Zr, V, Sn, Mo, Be, Ge, $\mathrm{Ni}$, and $\mathrm{Cr}$ were analyzed by the quantitative spectral method (Meyshtas, 1970) using the ISP-28 (U.S.S.R.). All samples were analyzed twice. Control of analysis quality was carried out by means of the Soviet geological standards SGD-1, SG-1, and ST-1, and standards of the German Democratic Republic. Intralaboratory geological standards were also used. Accuracy and quality of the analyses are thought to be good. Analyses were performed in the Laboratory of Atlantic Geology, Atlantic Branch, P.P. Shirshov Institute of Oceanology, Academy of Sciences of the U.S.S.R., under the author's guidance (analysts Yu. O. Shaydurov, G.S. Khandros, Z. Yablunovskaya, T.I. Khomina, and N.G. Kudryavtsev).

Silicate analyses were carried out by conventional chemical methods in the laboratory of the Geological Survey of Western Siberia (Novokuznetsk).

The comparative data on Recent and late Quaternary sediments near Leg 39 drill sites (Figure 1) is also included.

\section{CARBONATES}

$\mathrm{CaCO}_{3}$ content varies considerably, ranging from 0.0 up to $92.52 \%$ (Tables 1, 2, 3).

\section{Site 353}

The carbonate content in sediments from the Vema Fracture Zone is low. This is due to two reasons: (1) dilution of carbonate by terrigenous sediment from the Amazon River (Bader et al., 1970); and (2) depth of the site at or near the level of carbonate compensation depth (the compensation depth in the Guiana Basin is 5500 meters [Lisitzin, 1971; Emelyanov et al., 1975]).
Carbonates are mainly biogenic remains. Lowmagnesian calcite is the dominant mineral. Authigenic carbonates occur as single grains (Tables 4,5 ).

\section{Site 354}

$\mathrm{CaCO}_{3}$ content varies from 8.75 to $85.05 \%$. The minimum content of $\mathrm{CaCO}_{3}(8.75 \%)$ was found in the sediments of Pleistocene age as a result of strong dilution by terrigenous input from the Amazon River; carbonate content is considerably higher in the older sediments (37.02-85.05\%). Variations of $\mathrm{CaCO}_{3}$ content over such a wide range may be caused by one or more of the following: (1) variations of the rate of supply of biogenic carbonates to bottom sediments; (2) irregular supply of terrigenous material from the Amazon River drainage; (3) variations of the depth carbonate dissolution. The Ceará Rise site was presumably situated below the lysocline between late Eocene and middle Oligocene, a time when the lysocline might have been shallower than at present. After middle Oligocene, the content of $\mathrm{CaCO}_{3}$ increased slightly, which could be related to a subsidence of the lysocline or shoaling of the site. The high content of $\mathrm{CaCO}_{3}$ indicates that the site was above the carbonate compensation depth for most or all of the Tertiary.

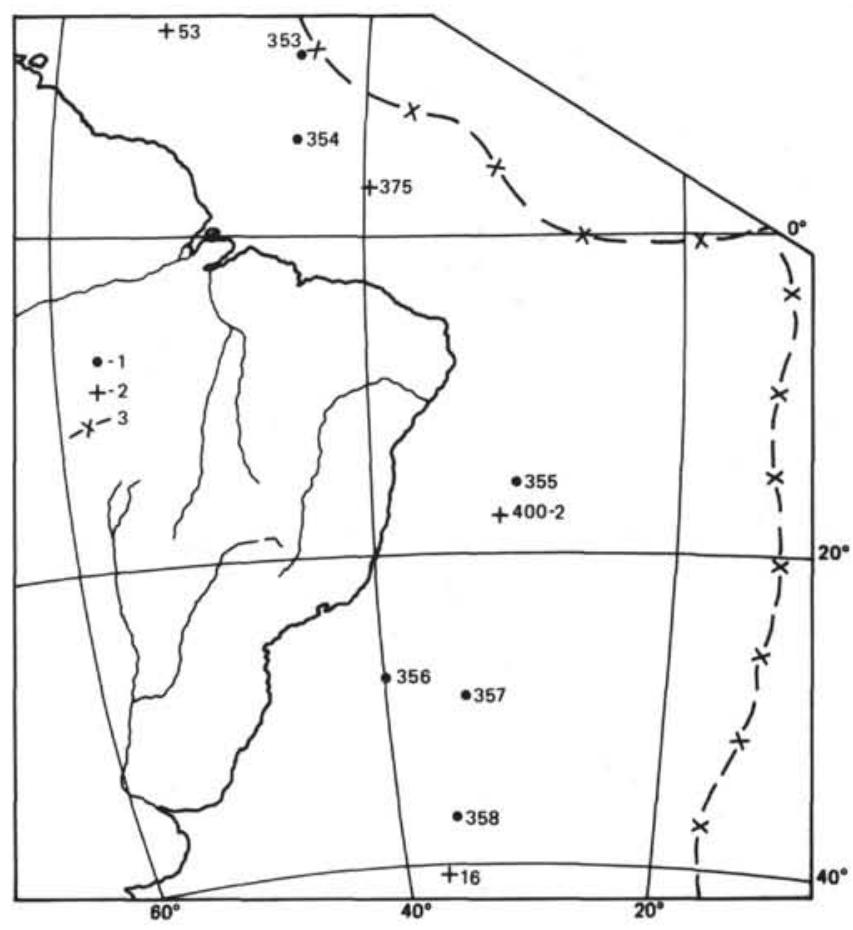

Figure 1. Location of DSDP and other sites. $1=$ Leg 39 sites, $2=$ other sites, $3=$ Mid Atlantic Ridge. 
TABLE 1

Range of Contents of Elements in the Sediments from Leg 39

\begin{tabular}{|c|c|c|c|c|c|c|c|c|c|}
\hline & & & & Content, $(9$ & & & & & \\
\hline Type of Sediments & $\mathrm{CaCO}_{3}$ & $\mathrm{C}_{\text {org }}$ & $\mathrm{Fe}$ & Mn & $\mathrm{Ti}$ & $\mathrm{P}$ & $\mathrm{Na}_{2} \mathrm{O}$ & $\mathrm{K}_{2} \mathrm{O}$ & $\mathrm{Zn}$ \\
\hline All types & $0.00-92.52$ & $0.03-1.35^{\mathrm{a}}$ & $\begin{array}{c}0.30-7.32 \\
(1.58-28.58)\end{array}$ & $\begin{array}{c}0.003-0.70 \\
(0.02-2.39)\end{array}$ & $\begin{array}{c}0.01-1.05 \\
(0.05-2.00)\end{array}$ & $\begin{array}{c}0.01-0.65 \\
(0.01-2.17)\end{array}$ & $0.37-3.10$ & $0.30-3.77$ & $\begin{array}{c}<4-231 \\
(<17-554)\end{array}$ \\
\hline
\end{tabular}

Note: Content given for dry sediment and for carbonate-free sediment (in brackets).

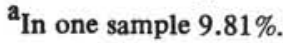

The carbonate consists mostly of coccoliths, with lesser foraminifers. Low magnesian calcite is the dominant mineral (Table 4). About $2 \%$ dolomite is found in sediments of Eocene, Oligocene, and Miocene age and 15 to $20 \%$ authigenic carbonate is present. Up to $6 \%$ dolomite and $4 \%$ siderite (Table 4 ) occur in the ferruginous marly nannofossil oozes.

\section{Site 355}

The site is below the present carbonate compensation depth $(4600-4800 \mathrm{~m})$ and is located in the zone of Recent red clays deposition in the Brazil Basin. Therefore, carbonate content is very low in the upper part of the core (lower Eocene-Pleistocene), which is characterized by terrigenous muds (and red clays) and by zeolitic pelagic muds. In the lower part of the core, in nannofossil oozes of Late Cretaceous age, the content of $\mathrm{CaCO}_{3}$ is high, being up to 60.79 to $89.12 \%$. On the basis of the smear slides study, the carbonate is represented by the remains of coccoliths $(40 \%$ on the average) and authigenic calcite (50\% on the average). Calcite occasionally forms hydrothermal veins with a thickness of 3-13 mm. These veins are associated with the brown-reddish ferruginous nannofossil muds. The degree of iron enrichment (oxidized form of $\mathrm{Fe}$ prevails; Table 3) increases with depth. Grains of dolomite were found in the upper part of the coccolith muds. On the basis of X-ray analyses calcite (only authigenic?) in the Cretaceous sediments contains 4-6 mol. $\% \mathrm{MgCO}_{3}$ in its lattice.

The carbonate data indicate that in the Late Cretaceous the bottom of the Brazil Basin (Site 355) was much shallower and was situated above the compensation depth. Site 355 in the Cretaceous was probably located within the limits of the Mid-Atlantic Ridge. In the early Maestrichtian the site subsided below the compensation depth (Sclater et al., 1971), and carbonate dissolution began.

\section{Site 356}

The content of $\mathrm{CaCO}_{3}$ varies in the range of 1.75 to $85.80 \%$ ( $40 \%$ on the average). The highest carbonate content was found in the sediments of early MiocenePleistocene age, from $48.78 \%$ up to $85.80 \%$. Carbonates are represented by coccoliths and foraminifers. In Units II and III (Core 16, Section 1 up to Core 6, Section 4), represented by siliceous-calcareous muds (coccoliths and foraminifers, mainly), the content of $\mathrm{CaCO}_{3}$ is 21.76 to $37.74 \%$ and 19.02 to $41.78 \%$, respectively. The dominant carbonate mineral is biogenic low-magnesian calcite. Dolomite is detected in the sediments of early Eocene age and authigenic calcite is present.

Unit IV (Core 26, Section 2 up to Core 19, Section 4) is represented by nannofossil muds (marly mud) with a $\mathrm{CaCO}_{3}$ content from 41.78 to $57.54 \%$. Interbeds of terrigenous and terrigenous-siliceous (?) muds with a low content of $\mathrm{CaCO}_{3}(14.76$ and $18.51 \%)$ are present in two cores of this unit (Core 17, Section 4 and Core 29, Section 2). Carbonates are represented by coccoliths, foraminifers, and authigenic calcite which contains about $5 \mathrm{~mol} . \% \mathrm{MgCO}_{3}$.

Unit V is represented by marly nannofossil muds (chalk), slightly cemented by authigenic calcite. It contains 39.02 to $45.28 \% \mathrm{CaCO}_{3}$. There is a considerable admixture of dolomite, increasing downward.

Unit VI (Core 40, Section 6 and Core 39, Section 5, clayey conglomerates and mudstones) contains low quantities of $\mathrm{CaCO}_{3}$, from 1.75 to $6.25 \%$. Carbonates are represented by coccoliths and authigenic calcite.

Unit VII (Core 44, Section 3 and Core 41, Section 3) consists of marly dolomitized limestones (32.02-37.27\% of $\left.\mathrm{CaCO}_{3}\right)$. The limestones are recrystallized carbonates (calcite) and are dolomitized. Coccoliths and foraminifers are in neglibible quantities.

Thus, judging from the carbonate studies, all sediments at Site 356 accumulated under pelagic conditions, but above the carbonate compensation depth. Active biogenic pelagic accumulation of carbonates took place (deposition of coccoliths, foraminifers) followed by the consequent recrystallization of these biogenic carbonates and diagenetic formation of calcite and dolomite.

\section{Site 357}

This site is on the Rio Grande Rise and the $\mathrm{CaCO}_{3}$ content in the sediments is high, up to $92.56 \%$. Although there are interlayers with very low content, from 1 to $3 \%$ (Table 2), at this site the depth was always moderate $(1000 \mathrm{~m}$ in the Cretaceous and $2100 \mathrm{~m}$ at present).

Unit I (foraminiferal-coccolith muds) is richest in $\mathrm{CaCO}_{3}$ being from 84.10 to $92.56 \%$. Planktonic foraminifers and coccoliths are dominant and pteropods and benthic foraminifers are found in negligible quantities.

The sediments of Unit II consist mainly of coccolith ooze and contain from 70.04 to $92.56 \% \mathrm{CaCO}_{3}$. It differs from the first unit by: (1) the presence of 
TABLE 1 - Continued

Content $\left(10^{-4} \%\right)$
$\mathrm{Cu}$
$\mathrm{Ni}$
Co
Cr
$\mathrm{Ba}$
$\mathrm{Zr}$
V
Mo
Be
$\mathrm{Ge} \quad \mathrm{Cd}$

$\begin{array}{ccccccccccc}6-232 & <6-354 & <4-65 & 7-513 & <200-1300 & <40-600 & 13-870 & <5-13 & <1-4 & <50 & <6 \\ (20-543) & (<13-864) & (<4-159) & (7-796) & (<200-3589) & (66-1138) & (46-883) & (<5-120) & (<1-8) & (<50-334) & <6\end{array}$

$<6-18 \quad<6-20 \quad<6-26 \quad 4-28$

TABLE 2

Chemical Composition of Sediments From Leg 39

\begin{tabular}{|c|c|c|c|c|c|c|c|c|c|c|c|c|c|c|}
\hline \multirow{2}{*}{$\begin{array}{c}\text { Sample } \\
\text { (Interval in cm) }\end{array}$} & \multicolumn{8}{|c|}{ (\%) } & \multicolumn{6}{|c|}{$\left(10^{-4} \%\right)$} \\
\hline & $\mathrm{CaCO}_{3}$ & $\mathrm{C}_{\text {org }}$ & $\mathrm{Fe}$ & Mn & $\mathrm{Ti}$ & $\mathbf{P}$ & $\mathrm{Na}_{2} \mathrm{O}$ & $\mathrm{K}_{2} \mathrm{O}$ & $\mathrm{Zn}$ & $\mathrm{Cu}$ & $\mathrm{Ni}$ & Co & $\mathrm{Cr}$ & $\mathrm{Cd}$ \\
\hline \multicolumn{15}{|l|}{ Hole 353} \\
\hline $2-2,30-32$ & 4.75 & 0.36 & 4.65 & 0.087 & 0.50 & 0.05 & 1.75 & 2.62 & 108 & 37 & 50 & 22 & 66 & 4.0 \\
\hline \multicolumn{15}{|l|}{ Hole 353A } \\
\hline $1-1,102-105$ & 4.25 & 0.48 & 5.30 & 0.070 & 0.47 & 0.05 & 1.76 & 2.84 & 120 & 19 & 48 & $<20$ & 42 & $<4.0$ \\
\hline \multicolumn{15}{|l|}{ Site 354} \\
\hline $1-1,115$ & 19.51 & 0.18 & 4.63 & 0.675 & 0.40 & 0.05 & 2.02 & 2.25 & 95 & 53 & 65 & .36 & 70 & 4.0 \\
\hline $1-2,119$ & 8.75 & 0.36 & 4.95 & 0.107 & 0.50 & 0.05 & 2.30 & 2.50 & 102 & 60 & 61 & 22 & 76 & $<4.0$ \\
\hline $3-1,120$ & 46.53 & 0.12 & 2.53 & 0.065 & 0.24 & 0.03 & 1.43 & 1.56 & 76 & 30 & 44 & 22 & 38 & 4.5 \\
\hline $4-1,40-42$ & 43.78 & 0.06 & 2.95 & 0.070 & 0.28 & 0.03 & 1.38 & 1.64 & 88 & 68 & 41 & 22 & 52 & $<4.0$ \\
\hline $4-2,40-42$ & 61.04 & 0.06 & 1.97 & 0.094 & 0.16 & 0.03 & 1.03 & 1.17 & 64 & 22 & 31 & 22 & 46 & $<4.0$ \\
\hline $5-2,71-73$ & 40.78 & 0.06 & 3.50 & 0.084 & 0.29 & 0.03 & 1.18 & 1.76 & 73 & 39 & 53 & 22 & 58 & $<4.0$ \\
\hline $6-3,91$ & 67,02 & 0.06 & 1.25 & 0.087 & 0.19 & 0.02 & 0.88 & 0.76 & 42 & 110 & 43 & $<20$ & 38 & $<4.0$ \\
\hline $6-2,107$ & 56.54 & 0.03 & 1.90 & 0.077 & 0.19 & 0.03 & 1.09 & 1.30 & 46 & 30 & 51 & $<20$ & 42 & $<4.0$ \\
\hline $7-1,137$ & 73.05 & 0.03 & 1.20 & 0.160 & 0.08 & 0.03 & 1.30 & 0.60 & 38 & 28 & 35 & $<20$ & 32 & $<4.0$ \\
\hline $7-3,16$ & 47.28 & 0.03 & 2.73 & 0.092 & 0.24 & 0.05 & 1.04 & 1.07 & 90 & 30 & 60 & $<20$ & 62 & $<4.0$ \\
\hline $8-2,30$ & 51.28 & 0.15 & 2.37 & 0.071 & 0.21 & 0.04 & 1.05 & 0.83 & 66 & 46 & 38 & $<20$ & 58 & $<4.0$ \\
\hline $9-3,112$ & 54.78 & 0.15 & 2.40 & 0.051 & 0.17 & 0.03 & 0.97 & 0.90 & 80 & 92 & 46 & 28 & 54 & $<4.0$ \\
\hline $10-2,98$ & 85.05 & 0.18 & 1.08 & 0.041 & 0.16 & 0.03 & 0.83 & 0.52 & 44 & 60 & 27 & $<20$ & 26 & $<4.0$ \\
\hline $11-5,25$ & 67.54 & 0.15 & 3.17 & 0.032 & 0.12 & 0.03 & 0.80 & 1.05 & 64 & 54 & 22 & 10 & 36 & $<4.0$ \\
\hline $12-4,11$ & 55.03 & 0.18 & 2.90 & 0.032 & 0.11 & 0.02 & 0.90 & 0.86 & 60 & 200 & 76 & 21 & 27 & $<4.0$ \\
\hline $13-6,125$ & 67.54 & 0.15 & 2.77 & 0.050 & 0.12 & 0.03 & 0.58 & 0.81 & 23 & 36 & 10 & 8 & 30 & $<4.0$ \\
\hline $14-1,106$ & 71.55 & 0.12 & 2.02 & 0.051 & 0.08 & 0.02 & 0.71 & 0.47 & 16 & 28 & 10 & 8 & 24 & $<4.0$ \\
\hline $15-2,45$ & 63.79 & 0.12 & 0.99 & 0.100 & 0.02 & 0.03 & 0.44 & 0.34 & 25 & 17 & 12 & 10 & 21 & $<4.0$ \\
\hline $15-3,84$ & 64.29 & 0.15 & 1.83 & 0.073 & 0.08 & 0.02 & 0.68 & 0.74 & 19 & 194 & 6 & 8 & 26 & $<4.0$ \\
\hline $17-2,117$ & 53.78 & 0.15 & 3.32 & 0.069 & 0.21 & 0.04 & 0.54 & 1.08 & 27 & 58 & 6 & 8 & 50 & $<4.0$ \\
\hline $18-1,130$ & 52.78 & 0.21 & 1.54 & 0.073 & 0.19 & 0.04 & 0.83 & 1.00 & 37 & 12 & 15 & 14 & 38 & $<4.0$ \\
\hline $18-4,128$ & 37.02 & 0.27 & 1.90 & 0.040 & 0.29 & 0.06 & 0.42 & 0.91 & 56 & 34 & 8 & 8 & 52 & $<4.0$ \\
\hline \multicolumn{15}{|l|}{ Site 355} \\
\hline $1-2,69-71$ & 0.25 & 0.24 & 5.47 & 0.27 & 0.63 & 0.05 & 2.32 & 2.65 & 14 & 88 & 75 & 35 & 53 & $<6$ \\
\hline $1-6,80-82$ & 1.00 & 0.27 & 5.33 & 0.67 & 0.56 & 0.04 & 2.54 & 2.54 & 138 & 85 & 72 & 20 & 54 & $<6$ \\
\hline $2-3,130-132$ & 1.25 & 0.30 & 5.54 & 0.30 & 0.59 & 0.05 & 2.06 & 2.82 & 132 & 84 & 56 & 25 & 52 & $<6$ \\
\hline $2-5,135-137$ & 0.75 & 0.21 & 6.01 & 0.10 & 0.52 & 0.06 & 1.88 & 3.34 & 146 & 76 & 84 & 20 & 50 & $<6$ \\
\hline $3-2,88-90$ & 8.50 & 0.51 & 4.91 & 0.13 & 0.48 & 0.05 & 2.11 & 2.26 & 130 & 56 & 75 & 15 & 54 & $<6$ \\
\hline $3-5,58-60$ & 0.75 & 0.54 & 5.55 & 0.24 & 0.59 & 0.05 & 2.14 & 2.35 & 152 & 94 & 86 & 31 & 44 & $<6$ \\
\hline $4-3,110-112$ & 0.00 & 0.36 & 6.07 & 0.09 & 0.67 & 0.06 & 1.53 & 2.24 & 166 & 73 & 78 & 25 & 69 & $<6$ \\
\hline $5-1,40-42$ & 0.50 & 0.39 & 5.32 & 0.08 & 0.59 & 0.06 & 1.88 & 2.21 & 135 & 46 & 56 & 15 & 54 & $<6$ \\
\hline $5-4,98-100$ & 0.24 & 0.45 & 4.65 & 0.15 & 0.56 & 0.04 & 2.11 & 2.35 & 111 & 33 & 57 & 15 & 55 & $<6$ \\
\hline $6-2,100-102$ & 5.00 & 0.63 & 5.63 & 0.07 & 0.59 & 0.05 & 2.12 & 2.21 & 123 & 42 & 45 & 15 & 54 & $<6$ \\
\hline $7-2,90-92$ & 2.50 & 0.30 & 5.14 & 0.05 & 0.59 & 0.05 & 1.90 & 2.40 & 122 & 40 & 107 & 31 & 63 & $<6$ \\
\hline $7-3,100-102$ & 4.50 & 0.51 & 6.26 & 0.18 & 0.63 & 0.06 & 1.70 & 2.18 & 145 & 33 & 56 & 15 & 64 & $<6$ \\
\hline $8-2,140-141$ & 0.00 & 0.48 & 3.54 & 0.37 & 0.46 & 0.06 & 2.13 & 1.84 & 106 & 32 & 72 & 25 & 61 & $<6$ \\
\hline $9-2,50-52$ & 0.00 & 0.39 & 3.62 & 0.02 & 0.52 & 0.03 & 1.61 & 1.76 & 104 & 232 & 52 & 21 & 60 & $<6$ \\
\hline $11-2,91-92$ & 0.00 & 0.21 & 4.52 & 0.04 & 0.50 & 0.04 & 1.44 & 1.67 & 125 & 24 & 66 & 23 & 87 & $<6$ \\
\hline $12-4,60-62$ & 0.00 & 0.42 & 4.65 & 0.02 & 0.48 & 0.03 & 1.40 & 1.52 & 138 & 26 & 86 & 25 & 79 & $<6$ \\
\hline $13-3,52-54$ & 0.00 & 0.33 & 4.73 & 0.04 & 0.52 & 0.03 & 1.44 & 1.81 & 134 & 85 & 82 & 23 & 82 & $<6$ \\
\hline $14-3,93-94$ & 0.00 & 0.27 & 5.37 & 0.04 & 0.59 & 0.05 & 1.36 & 2.24 & 157 & 50 & 100 & 37 & 88 & $<6$ \\
\hline
\end{tabular}


TABLE 2 - Continued

$(\%)$ $\left(10^{-4} \%\right)$

Sample

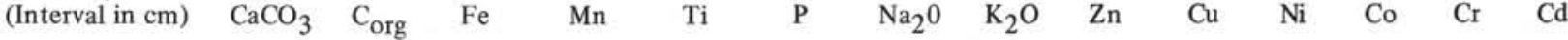

\begin{tabular}{lrrrrrrrrrrrrrr}
\hline $14-5,87-89$ & 0.00 & 0.30 & 6.77 & 0.07 & 0.61 & 0.06 & 1.33 & 2.35 & 156 & 30 & 96 & 32 & 93 & $<6$ \\
$15-1,84$ & 1.50 & 0.63 & 4.53 & 0.70 & 0.63 & 0.05 & 1.30 & 2.86 & 171 & 113 & 86 & 33 & 93 & $<6$ \\
$15-1,127$ & 0.00 & 0.51 & 7.32 & 0.13 & 0.65 & 0.06 & 1.19 & 2.64 & 160 & 27 & 112 & 37 & 93 & $<6$ \\
$17-3,140-142$ & 69.80 & 0.30 & 1.21 & 0.06 & 0.14 & 0.03 & 0.60 & 1.08 & 45 & 6 & 19 & 11 & 23 & $<6$ \\
$17-4,15-17$ & 75.05 & 0.36 & 1.76 & 0.12 & 0.12 & 0.03 & 0.58 & 0.96 & 26 & 9 & 31 & 10 & 21 & $<6$ \\
$17-6,61-63$ & 81.30 & 0.39 & 1.18 & 0.10 & 0.03 & 0.02 & 0.52 & 0.73 & 18 & 8 & 21 & 10 & 18 & $<6$ \\
$18-1,115-117$ & 75.78 & 0.51 & 1.23 & 0.15 & 0.08 & 0.02 & 0.62 & 0.65 & 22 & 21 & 26 & 10 & 18 & $<6$ \\
$18-3,36-38$ & 64.79 & 0.15 & 2.05 & 0.18 & 0.16 & 0.03 & 0.58 & 1.30 & 52 & 30 & 24 & 16 & 24 & $<6$ \\
$19-2,127-129$ & 60.79 & 0.66 & 3.08 & 0.76 & 0.23 & 0.06 & 0.82 & 1.53 & 66 & 44 & 69 & 36 & 30 & $<6$ \\
$20-2,115-117$ & 89.12 & 0.48 & 1.40 & 0.26 & 0.08 & 0.04 & 0.49 & 0.68 & 24 & 23 & 28 & 10 & 13 & $<6$
\end{tabular}

Hole 356

\begin{tabular}{|c|c|c|c|c|c|c|c|c|c|c|c|c|c|c|}
\hline $2-1,28-31$ & 85.80 & 1.08 & 0.70 & 0.03 & 0.05 & 0.03 & 1.02 & 0.34 & 18 & 20 & 12 & 10 & 15 & $<6$ \\
\hline $3-2,71-73$ & 57.54 & 0.51 & 2.05 & 0.05 & 0.23 & 0.05 & 2.08 & 1.15 & 56 & 20 & 9 & $<10$ & 29 & $<6$ \\
\hline $4-3,43-45$ & 48.78 & 1.08 & 2.29 & 0.05 & 0.23 & 0.04 & 2.22 & 1.33 & 70 & 20 & 11 & $<10$ & 33 & $<6$ \\
\hline $5-4,120-123$ & 60.04 & 1.02 & 1.52 & 0.06 & 0.18 & 0.04 & 2.10 & 1.01 & 59 & 44 & 11 & $<10$ & 18 & $<6$ \\
\hline $6-4,80-83$ & 25.52 & 0.51 & 2.95 & 0.06 & 0.27 & 0.03 & 2.18 & 1.57 & 71 & 25 & 16 & $<10$ & 44 & $<6$ \\
\hline $7-4,30-33$ & 21.76 & 0.66 & 3.18 & 0.04 & 0.31 & 0.05 & 2.08 & 1.60 & 74 & 27 & 19 & $<10$ & 47 & $<6$ \\
\hline $8-2,70-73$ & 30.02 & 0.75 & 3.24 & 0.04 & 0.42 & 0.03 & 1.95 & 1.71 & 85 & 28 & 19 & $<10$ & 37 & $<6$ \\
\hline $9-2,130-132$ & 37.77 & 0.54 & 3.00 & 0.08 & 0.27 & 0.04 & 1.70 & 1.40 & 52 & 23 & 6 & $<10$ & 35 & $<6$ \\
\hline $10-3,59-61$ & 29.52 & 1.14 & 2.46 & 0.12 & 0.37 & 0.03 & 2.36 & 2.04 & 98 & 34 & 22 & $<10$ & 57 & $<6$ \\
\hline $11-3,113-115$ & 41.78 & 0.81 & 3.00 & 0.13 & 0.27 & 0.05 & 1.62 & 1.37 & 56 & 13 & 11 & $<10$ & 45 & $<6$ \\
\hline $12-1,9-11$ & 19.01 & 0.48 & 2.57 & 0.08 & 0.31 & 0.03 & 1.62 & 1.33 & 56 & 23 & 24 & 13 & 47 & $<6$ \\
\hline $14-1,20-23$ & 31.52 & 0.54 & 1.81 & 0.13 & 0.20 & 0.03 & 1.41 & 0.98 & 62 & 50 & 13 & $<10$ & 35 & $<6$ \\
\hline $16-1,127-129$ & 22.26 & 0.72 & 2.64 & 0.12 & 0.27 & 0.03 & 1.36 & 1.14 & 96 & 33 & 26 & $<10$ & 55 & $<6$ \\
\hline $17-4,60-63$ & 14.76 & 0.60 & 3.86 & 0.04 & 0.52 & 0.03 & 1.95 & 2.22 & 116 & 73 & 58 & 13 & 70 & $<-"-$ \\
\hline $19-4,42-44$ & 41.78 & 0.78 & 2.00 & 0.09 & 0.25 & 0.03 & 1.27 & 1.68 & 38 & 34 & 75 & 20 & 29 & $<-"-$ \\
\hline $23-2,60-62$ & 42.53 & 0.90 & 2.78 & 0.10 & 0.37 & 0.03 & 1.55 & 1.71 & 64 & 31 & 13 & $<10$ & 45 & $<6$ \\
\hline $24-5,121-123$ & 45.54 & 0.60 & 3.25 & 0.12 & 0.29 & 0.06 & 1.31 & 1.73 & 82 & 20 & 22 & $<10$ & 42 & $<6$ \\
\hline $25-2,40-42$ & 57.54 & 1.20 & 2.52 & 0.13 & 0.27 & 0.03 & 1.24 & 1.50 & 71 & 18 & 19 & $<10$ & 33 & $<6$ \\
\hline $26-2,26-28$ & 52.31 & 1.20 & 3.01 & 0.14 & 0.30 & 0.03 & 1.21 & 1.74 & 75 & 45 & 22 & $<10$ & 32 & $<6$ \\
\hline $29-2,120-122$ & 18.51 & 0.60 & 3.88 & 0.04 & 0.50 & 0.03 & 1.80 & 2.68 & 93 & 32 & 45 & 25 & 63 & $<6$ \\
\hline $29-6,40-42$ & 55.03 & 0.69 & 2.12 & 0.16 & 0.23 & 0.03 & 1.15 & 1.57 & 57 & 23 & 9 & $<10$ & 29 & $<6$ \\
\hline $31-5,54-56$ & 42.03 & 0.66 & 2.60 & 0.07 & 0.31 & 0.04 & 1.17 & 2.00 & 56 & 23 & 16 & $<10$ & 31 & $<6$ \\
\hline $33-2,88-90$ & 39.02 & 0.60 & 3.50 & 0.07 & 0.35 & 0.03 & 1.20 & 2.20 & 70 & 22 & 13. & $<10$ & 50 & $<-"-$ \\
\hline $35-3,70-72$ & 42.03 & 1.05 & 2.57 & 0.10 & 0.34 & 0.05 & 1.35 & 1.79 & 77 & 23 & 16 & $<10$ & 46 & $<6$ \\
\hline $38-3,107-109$ & 45.28 & 0.75 & 3.27 & 0.09 & 0.48 & 0.05 & 0.90 & 2.01 & 65 & 29 & 24 & $<10$ & 55 & $<6$ \\
\hline $39-5,95-97$ & 6.25 & 0.72 & 5.66 & 0.032 & 0.79 & 0.05 & 1.30 & 3.66 & 86 & 78 & 42 & 12 & 92 & $<6$ \\
\hline $40-6,44-46$ & 1.75 & 1.35 & 4.34 & 0.021 & 0.65 & 0.05 & 1.39 & 2.62 & 130 & 85 & 25 & 13 & 111 & $<6$ \\
\hline $41-4,56-58$ & 32.02 & 9.81 & 3.41 & 0.03 & 1.01 & 0.02 & 1.01 & 1.57 & 109 & 95 & 208 & $<10$ & 60 & 15 \\
\hline $44-3,109-111$ & $37-77$ & 0.87 & 3.32 & 0.10 & 0.40 & 0.05 & 1.21 & 2.06 & 52 & 33 & 14 & $<10$ & 37 & $<6$ \\
\hline
\end{tabular}

Hole 356A

$1-4,74-76$

$2-5,73-75$

$\begin{array}{lll}33.02 & 0.36 & 2.86\end{array}$

0.03

0.3

$2.48 \quad 1.60 \quad 61$

$27 \quad 9<10 \quad 41<6$

Site 357

$1-3,93-96$
$3-2,97-99$
$5-3,90-92$
$6-4,70-73$
$8-2,80-83$
$9-2,60-63$
$10-1,70-72$
$10-2,70-72$
$11-2,108-111$
$13-5,65-68$
$15-2,100-103$
$17-2,70-72$
$20-3,91-94$
$22-3,84-87$
$23-3,42-45$
$24-1,96-99$
$24-5,75-78$

$\begin{array}{llllllll}92.56 & 0.45 & 0.40 & 0.03 & 0.006 & 0.02 & 0.94 & 0.19 \\ 89.56 & 0.42 & 0.34 & 0.03 & 0.01 & 0.01 & 1.16 & 0.22 \\ 84.10 & 0.84 & 0.95 & 0.03 & 0.08 & 0.02 & 1.50 & 0.53 \\ 85.30 & 0.75 & 0.78 & 0.04 & 0.05 & 0.03 & 0.95 & 0.54 \\ 87-81 & 0.36 & 0.47 & 0.02 & 0.01 & 0.03 & 1.10 & 0.33 \\ 92.56 & 0.30 & 0.35 & 0.003 & 0.01 & 0.03 & 0.85 & 0.21 \\ 84.30 & 0.24 & 0.49 & 0.02 & 0.03 & 0.03 & 0.86 & 0.77 \\ 81.06 & 0.69 & 0.30 & 0.02 & 0.01 & 0.03 & 1.04 & 0.27 \\ 89-06 & 0.60 & 0.37 & 0.03 & 0.01 & 0.03 & 0.99 & 0.36 \\ 80.30 & 0.54 & 0.74 & 0.03 & 0.04 & 0.05 & 1.29 & 0.66 \\ 81.55 & 0.60 & 0.81 & 0.03 & 0.08 & 0.06 & 0.96 & 0.66 \\ 76.30 & 0.54 & 0.88 & 0.02 & 0.10 & 0.05 & 1.14 & 0.83 \\ 86.05 & 0.27 & 0.98 & 0.02 & 0.08 & 0.05 & 0.38 & 0.57 \\ 81.30 & 0.54 & 0.98 & 0.04 & 0.10 & 0.04 & 0.87 & 0.59 \\ 73-87 & 0.30 & 1.36 & 0.05 & 0.31 & 0.07 & 0.85 & 0.49 \\ 85.56 & 0.33 & 0.60 & 0.05 & 0.08 & 0.04 & 0.86 & 0.62 \\ 64.29 & 0.39 & 1.50 & 0.05 & 0.61 & 0.12 & 1.36 & 0.64\end{array}$

4
4
14
15
7
25
24
6
8
16
14
25
8
35
23
80
66

$\begin{array}{rrrrr}17 & <8 & <10 & 10 & <6 \\ 14 & <8 & <10 & 11 & <6 \\ 14 & 9 & <10 & 13 & <6 \\ 16 & 9 & <10 & 17 & <6 \\ 11 & <8 & <10 & 12 & <6 \\ 17 & <8 & 5 & 14 & <6 \\ 14 & <8 & 6 & 14 & <6 \\ 13 & <8 & 9 & 12 & <6 \\ 14 & <8 & 12 & 16 & <6 \\ 15 & 8 & <4 & 17 & <6 \\ 15 & 8 & 8 & 19 & <6 \\ 18 & <8 & <4 & 19 & <6 \\ 5 & <8 & <4 & 17 & <6 \\ 16 & 8 & 4 & 35 & <6 \\ 17 & 9 & <10 & 90 & <6 \\ 14 & 9 & <10 & 24 & <6 \\ 60 & 58 & 13 & 154 & <6\end{array}$


TABLE 2 - Continued

(\%) $\left(10^{-4} \%\right)$

Sample

\begin{tabular}{|c|c|c|c|c|c|c|c|c|c|c|c|c|c|c|}
\hline (Interval in $\mathrm{cm}$ ) & $\mathrm{CaCO}_{3}$ & $\mathrm{C}_{\text {org }}$ & $\mathrm{Fe}$ & $\mathrm{Mn}$ & $\mathrm{Ti}$ & $\mathrm{P}$ & $\mathrm{Na}_{2} \mathrm{O}$ & $\mathrm{K}_{2} \mathrm{O}$ & $\mathrm{Zn}$ & $\mathrm{Cu}$ & $\mathrm{Ni}$ & Co & $\mathrm{Cr}$ & $\mathrm{Cd}$ \\
\hline $24-5,84-87$ & 35.52 & 0.60 & 2.08 & 0.02 & 1.05 & 0.20 & 1.96 & 0.85 & 29 & 20 & 37 & $<10$ & 513 & $<6$ \\
\hline $24-5,91-94$ & 59.04 & 0.72 & 2.68 & 0.07 & 0.80 & & 1.48 & 0.86 & 33 & 45 & 354 & 65 & 224 & $<6$ \\
\hline $24-6,101-104$ & 89.56 & 0.54 & 2.23 & 0.05 & 0.10 & 0.03 & 0.37 & 0.32 & 14 & 11 & 31 & $<10$ & 16 & $<6$ \\
\hline $24-6,131-134$ & 90.31 & 0.51 & 2.77 & 0.07 & 0.16 & 0.03 & 0.62 & 0.45 & 27 & 8 & 29 & $<10$ & 32 & $<6$ \\
\hline $26-3,148-151$ & 70.80 & 0.30 & 1.19 & 0.04 & 0.22 & 0.06 & 0.65 & 0.52 & 14 & 15 & 9 & $<10$ & 35 & $<6$ \\
\hline $27-3,107-110$ & 70.04 & 0.60 & 1.45 & 0.06 & 0.35 & 0.65 & 1.54 & 0.97 & 16 & 16 & 18 & 8 & 19 & $<6$ \\
\hline $28-1,92-95$ & 24.27 & 0.39 & 3.48 & 0.04 & 0.79 & 0.19 & 1.92 & 1.15 & 72 & 20 & 33 & 12 & 20 & $<6$ \\
\hline $29-1,6-9$ & 87.05 & 0.24 & 0.59 & 0.06 & 0.05 & 0.03 & 1.23 & 0.83 & 26 & 14 & 15 & 5 & 22 & $<6$ \\
\hline $30-1,13-16$ & 83.30 & 0.33 & 0.92 & 0.04 & 0.08 & 0.03 & 0.74 & 0.72 & 14 & 17 & 21 & 4 & 16 & $<6$ \\
\hline $31-2,122-125$ & 80.80 & 0.63 & 0.62 & 0.05 & 0.08 & 0.01 & 0.70 & 0.90 & 9 & 16 & 12 & 7 & 19 & $<6$ \\
\hline $33-3,140-143$ & 63.04 & 0.36 & 2.00 & 0.64 & 0.23 & 0.03 & 1.05 & 1.79 & 35 & 18 & 23 & 6 & 28 & $<6$ \\
\hline $34-4,34-37$ & 51.28 & 0.48 & 1.79 & 0.06 & 0.23 & 0.03 & 1.14 & 2.10 & 50 & 23 & 23 & 5 & 28 & $<6$ \\
\hline $36-1,65-68$ & 44.03 & 0.24 & 3.12 & 0.06 & 0.30 & 0.03 & - & - & 68 & 20 & 24 & 6 & 39 & $<6$ \\
\hline $40-1,76-79$ & 55.79 & 0.36 & 2.38 & 0.06 & 0.31 & 0.03 & - & 1.91 & 76 & 28 & 20 & $<4$ & 32 & $<6$ \\
\hline $40-4,44-45$ & 1.00 & 0.39 & 3.41 & 0.02 & 0.34 & 0.01 & 2.06 & 0.67 & 160 & 18 & 34 & 50 & 9 & $<6$ \\
\hline $41-2,76-79$ & 15.01 & 0.24 & 3.59 & 0.02 & 0.34 & 0.02 & 1.81 & 1.11 & 111 & 16 & 85 & 18 & 19 & $<6$ \\
\hline $41-2,81-82$ & 3.00 & 0.39 & 4.67 & 0.003 & 0.31 & 0.03 & 1.92 & 0.47 & 231 & 19 & 520 & 75 & 7 & $<6$ \\
\hline $42-3,37-40$ & 40.78 & 0.42 & 3.02 & 0.05 & 0.40 & 0.03 & 1.12 & 2.48 & 101 & 37 & 38 & 11 & 47 & $<6$ \\
\hline $43-3,82-85$ & 56.04 & 0.36 & 1.82 & 0.05 & 0.27 & 0.03 & 0.74 & 1.57 & 51 & 27 & 26 & 6 & 32 & $<6$ \\
\hline $44-3,30-33$ & 47.28 & 0.57 & 3.82 & 0.05 & 0.41 & 0.03 & 1.02 & 2.30 & 127 & 32 & 61 & $<4$ & 51 & $<6$ \\
\hline $46-3,72-75$ & 55.54 & 0.51 & 3.05 & 0.05 & 0.34 & 0.04 & 0.85 & 2.04 & 80 & 32 & 33 & $<4$ & 34 & $<6$ \\
\hline $47-2,60-63$ & 59.54 & 0.36 & 2.33 & 0.06 & 0.29 & 0.05 & 0.90 & 1.94 & 65 & 33 & 31 & 8 & 32 & $<6$ \\
\hline $48-1,42-43$ & 67.29 & 0.39 & 1.30 & 0.06 & 0.16 & 0.03 & - & - & 25 & 20 & 15 & $<4$ & 26 & $<6$ \\
\hline $50-2,126-128$ & 48.53 & 0.72 & 1.45 & 0.06 & 0.14 & 0.03 & 0.65 & 0.88 & 34 & 18 & 7 & 5 & 22 & $<6$ \\
\hline $51-3.34-36$ & 40.27 & 0.45 & 1.34 & 0.05 & 0.16 & - & 0.76 & 0.88 & 10 & 19 & 13 & $<4$ & 20 & $<6$ \\
\hline
\end{tabular}

Site 358

$\begin{array}{lrrrrl}1-6,82-84 & 0.00 & 0.48 & 4.86 & 0.06 & 0.52 \\ 2-4,94-96 & 0.00 & 0.45 & 4.43 & 0.09 & 0.50 \\ 3-2,68-70 & 0.00 & 0.24 & 5.48 & 0.06 & 0.53 \\ 3-5,68-70 & 0.00 & 0.54 & 5.28 & 0.18 & 0.52 \\ 4-1,98-100 & 0.00 & 0.18 & 4.26 & 0.06 & 0.42 \\ 5-1,56-58 & 0.00 & 0.18 & 4.04 & 0.04 & 0.45 \\ 6-3,71-73 & 0.00 & 0.24 & 4.06 & 0.06 & 0.47 \\ 7-1,69-71 & 0.00 & 0.18 & 4.43 & 0.04 & 0.48 \\ 8-1,112-114 & 0.00 & 0.33 & 4.04 & 0.04 & 0.50 \\ 9-3,28-30 & 0.00 & 0.24 & 4.09 & 0.08 & 0.50 \\ 10-4,68-70 & 0.00 & 0.39 & 4.00 & 0.03 & 0.47 \\ 11-3,9-11 & 0.00 & 0.30 & 4.58 & 0.07 & 0.50 \\ 11-4,82-84 & 61.29 & 0.24 & 1.60 & 0.03 & 0.16 \\ 12-3,77-79 & 71.04 & 0.30 & 1.10 & 0.30 & 0.12 \\ 12-4,61-63 & 40.02 & 0.24 & 2.45 & 0.19 & 0.22 \\ 13-4,105-107 & 22.01 & 0.45 & 4.44 & 0.12 & 0.38 \\ 14-2,138-140 & 40.02 & 0.30 & 3.62 & 0.18 & 0.38 \\ 15-1,65-67 & 1.75 & 0.36 & 5.40 & 0.10 & 0.56 \\ 16-2,101-103 & 10.26 & 0.33 & 4.63 & 0.12 & 0.46\end{array}$

$\begin{array}{lllrrrrrr}0.03 & 2.76 & 2.74 & 122 & 107 & 38 & 22 & 43 & <6 \\ 0.02 & 3.10 & 2.48 & 108 & 82 & 46 & 24 & 45 & <6 \\ 0.02 & 2.41 & 3.00 & 108 & 119 & 68 & 30 & 38 & <6 \\ 0.11 & 2.35 & 2.78 & 102 & 74 & 46 & 22 & 38 & <6 \\ 0.07 & 2.99 & 2.46 & 88 & 32 & 50 & 22 & 31 & <6 \\ 0.03 & 3.13 & 2.74 & 94 & 65 & 38 & 13 & 36 & <6 \\ 0.03 & 2.90 & 2.43 & 140 & 83 & 48 & 10 & 33 & <6 \\ 0.03 & 2.75 & 2.56 & 97 & 54 & 33 & 11 & 33 & <6 \\ 0.03 & 2.07 & 2.76 & 196 & 78 & 33 & 13 & 50 & <6 \\ 0.03 & 1.85 & 3.23 & 111 & 70 & 27 & 5 & 44 & <6 \\ 0.05 & 1.82 & 3.22 & 118 & 49 & 44 & 4 & 47 & <6 \\ 0.08 & 2.04 & 1.94 & 113 & 39 & 52 & 13 & 31 & <6 \\ 0.03 & 0.96 & 1.08 & 56 & 23 & 35 & 11 & 20 & <6 \\ 0.03 & 0.85 & 0.83 & 37 & 15 & 28 & 6 & 25 & <6 \\ 0.05 & 1.14 & 1.38 & 69 & 76 & 30 & 11 & 44 & <6 \\ 0.07 & 1.40 & 3.10 & 123 & 64 & 46 & 18 & 45 & <6 \\ 0.08 & 1.36 & 2.45 & 108 & 42 & 44 & <6 & 45 & <6 \\ 0.05 & 1.67 & 3.77 & 128 & 82 & 57 & 22 & 38 & <6 \\ 0.05 & 1.51 & 3.09 & 95 & 40 & 32 & 18 & 42 & <6\end{array}$

Hole 359

\begin{tabular}{|c|c|c|c|c|c|c|c|c|c|c|c|c|c|c|}
\hline $1-3,72-74$ & 89.31 & 0.15 & 0.12 & 0.01 & 0.006 & 0.01 & 1.11 & 0.07 & $<2$ & 12 & $<8$ & $<6$ & 10 & $<6$ \\
\hline $32-134$ & 71.80 & 0.18 & 2.52 & 0.12 & 0.23 & 0.18 & 1.45 & 0.72 & 30 & 17 & 20 & $<6$ & 27 & \\
\hline $3-4,66-68$ & 3.25 & 0.24 & 3.98 & 0.06 & 0.25 & 0.02 & 4.73 & 4.38 & 223 & 6 & 10 & $<6$ & 6 & 6 \\
\hline $3-5,49-51$ & 56.29 & 0.24 & 2.64 & 0.11 & 0.52 & 0.08 & 2.19 & 1.53 & 52 & 18 & 10 & $<6$ & 28 & \\
\hline$-2,17-19$ & 7.90 & 0.39 & 6.07 & 0.15 & 1.58 & 0.26 & 1.95 & 1.01 & 133 & 7 & 26 & 26 & 4 & \\
\hline
\end{tabular}

Hole 359A

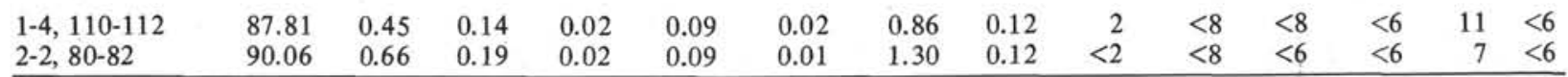

authigenic calcite, the quantity of which increases downward; (2) lesser quantity of foraminifer shells; and (3) the absence of pteropods. The oozes are locally cemented, grading into limestones. Nannofossil oozes with a decreased content of $\mathrm{CaCO}_{3}$ from 35.52 to $64.29 \%$ (Core 24, Section 5) are typical of the second unit. These sediments are enriched by silica (spicules, diatoms, radiolaria) and volcanic material (weathered ash). An interlayer at the very bottom of the unit (Core 28 , Section 1) contains $24.27 \%$ of $\mathrm{CaCO}_{3}$. These are silico-terrigenous sediments.

Unit III was not studied, but is similar to Unit II. 
TABLE 3

Total Chemical Composition of the Sediments in the Central Atlantic, Leg 39 (in \%)

\begin{tabular}{|c|c|c|c|c|c|c|c|c|c|c|c|c|c|c|c|c|}
\hline $\begin{array}{l}\text { Sample } \\
\text { (Interval in } \mathrm{cm} \text { ) }\end{array}$ & $\mathrm{SiO}_{2}$ & $\mathrm{TiO}_{2}$ & $\mathrm{Al}_{2} \mathrm{O}_{3}$ & $\mathrm{FeO}$ & $\mathrm{Fe}_{2} \mathrm{O}_{3}$ & $\mathrm{MnO}$ & $\mathrm{CaO}$ & $\mathrm{MgO}$ & $\mathrm{K}_{2} \mathrm{O}$ & $\mathrm{Na}_{2} \mathrm{O}$ & $\mathrm{P}_{2} \mathrm{O}_{5}$ & $\mathrm{CO}_{2}$ & L. o. i. & $\mathrm{S}$ & $\mathrm{F}$ & $\mathrm{H}_{2} \mathrm{O}^{+}$ \\
\hline $353-2-2,30-32$ & 52.72 & 0.92 & 19.36 & 1.73 & 5.35 & 0.06 & 3.11 & 2.84 & 2.69 & 1.69 & 0.217 & 2.98 & 9.45 & 0.15 & 0.09 & 6.64 \\
\hline $353-3-2,120-122$ & 78.76 & 0.48 & 7.35 & 4.03 & 1.51 & 0.04 & 1.41 & 1.02 & 1.50 & 1.36 & 0.068 & 0.61 & 1.90 & 0.17 & 0.02 & 1.74 \\
\hline $354-8-2,30$ & 26.19 & 0.50 & 11.42 & 0.29 & 3.77 & 0.03 & 22.44 & 1.22 & 0.98 & 1.27 & 0.142 & 21.47 & 26.50 & 0.17 & 0.06 & 5.02 \\
\hline $354-14-1,106$ & 14.94 & 0.30 & 4.48 & 0.43 & 1.79 & 0.03 & 41.16 & 1.22 & 0.31 & 1.02 & 0.093 & 31.64 & 34.03 & 0.14 & 0.04 & 1.80 \\
\hline $354-18-4,127$ & 40.75 & 0.58 & 12.19 & 1.73 & 1.96 & 0.05 & 18.93 & 1.22 & 1.00 & 0.41 & 0.235 & 16.50 & 20.49 & 0.22 & 0.05 & 4.22 \\
\hline $355-1-2,72-74$ & 49.90 & 1.07 & 19.24 & 0.43 & 8.26 & 0.06 & 1.13 & 3.45 & 2.82 & 2.82 & 0.223 & 0.45 & 9.24 & 1.28 & 0.06 & 8.34 \\
\hline $355-3-5,60-62$ & 52.86 & 1.11 & 19.24 & 0.43 & 8.82 & 0.06 & 1.13 & 3.65 & 2.58 & 2.42 & 0.247 & 2.32 & 8.10 & 0.20 & 0.09 & 7.20 \\
\hline $355-5-1,38-40$ & 55.07 & 1.07 & 17.87 & 0.29 & 7.64 & 0.06 & 0.58 & 2.94 & 2.46 & 2.32 & 0.223 & 0.00 & 9.05 & 0.16 & 0.09 & 9.10 \\
\hline $355-5-4,100-102$ & 56.09 & 1.08 & 17.69 & 0.43 & 7.10 & 0.06 & 0.58 & 3.45 & 2.50 & 2.14 & 0.167 & 0.00 & 8.25 & 0.23 & 0.08 & 8.20 \\
\hline $355-12-5,86-88$ & 60.69 & 1.02 & 15.92 & 1.15 & 6.63 & 0.06 & 0.58 & 2.84 & 2.12 & 1.32 & 0.161 & 0.91 & 6.93 & 0.29 & 0.06 & 6.09 \\
\hline $355-13-3,75-77$ & 55.01 & 1.04 & 17.97 & 0.43 & 9.46 & 0.06 & 0.58 & 3.25 & 2.32 & 1.33 & 0.124 & 0.00 & 8.02 & 0.08 & 0.08 & 8.00 \\
\hline $355-17-4,20-22$ & 12.36 & 0.34 & 4.30 & 0.14 & 3.24 & 0.03 & 41.27 & 1.42 & 1.13 & 1.02 & 0.093 & 32.77 & 34.52 & 0.06 & 0.05 & 1.98 \\
\hline $356-5-4,101-103$ & 23.63 & 0.45 & 6.28 & 0.21 & 2.19 & 0.03 & 32.23 & 1.63 & 1.13 & 2.72 & 0.198 & 26.89 & 29.17 & 0.14 & 0.06 & 2.74 \\
\hline $356-6-4,50-53$ & 43.82 & 0.72 & 9.34 & 0.43 & 4.15 & 0.05 & 15.46 & 2.84 & 1.77 & 2.40 & 0.173 & 12.88 & 18.47 & 0.27 & 0.08 & 5.49 \\
\hline $356-14-1,25-27$ & 49.64 & 0.44 & 6.91 & 0.36 & 2.64 & 0.05 & 17.16 & 2.03 & 1.13 & 1.62 & 0.198 & 13.41 & 17.40 & 0.11 & 0.08 & 4.00 \\
\hline $356-24-5,120-122$ & 31.56 & 0.62 & 9.24 & 0.43 & 4.64 & 0.04 & 24.12 & 2.44 & 1.88 & 1.69 & 0.266 & 18.76 & 23.00 & 0.12 & 0.07 & 4.12 \\
\hline $356-39-5,75-77$ & 51.46 & 1.43 & 13.55 & 2.01 & 6.77 & 0.07 & 0.85 & 4.27 & 3.42 & 1.52 & 0.266 & 2.71 & 10.34 & 1.69 & 0.09 & 7.74 \\
\hline $357-40-1,76-79$ & 26.24 & 0.65 & 7.17 & 1.15 & 2.77 & 0.04 & 28.64 & 3.25 & 2.00 & 1.27 & 0.148 & 23.73 & 26.35 & 0.19 & 0.08 & 2.76 \\
\hline $357-51-3,34-36$ & 48.85 & 0.39 & 4.12 & 0.54 & 1.50 & 0.05 & 21.20 & 2.24 & 0.88 & 0.88 & 0.148 & 16.16 & 18.91 & 0.35 & 0.07 & 2.80 \\
\hline $357-9-2,60-63$ & 2.23 & 0.16 & 1.08 & 0.43 & 0.18 & 0.02 & 52.01 & 0.81 & 0.26 & 1.44 & 0.179 & 41.81 & 41.09 & 0.07 & 0.04 & 0.49 \\
\hline $358-1-6,70-72$ & 54.69 & 1.02 & 17.76 & 1.13 & 6.42 & 0.06 & 0.85 & 3.64 & 2.95 & 2.35 & 0.137 & tr & 8.24 & 0.31 & 0.05 & 8.30 \\
\hline $358-3-2,71-73$ & 55.51 & 1.08 & 17.76 & 0.94 & 6.69 & 0.06 & 0.85 & 3.75 & 3.20 & 2.60 & 0.105 & $\mathrm{tr}$ & 7.00 & 0.17 & 0.09 & 6.98 \\
\hline $358-3-5,71-73$ & 55.91 & 1.09 & 17.44 & 0.58 & 7. & 0.06 & 0.85 & 3.75 & 2.90 & 2.70 & 0.334 & tr & 6.50 & 0.07 & 0.08 & 6.58 \\
\hline $358-5-2,62-64$ & 56.92 & 0.99 & 15.92 & 0.58 & 6.12 & 0.06 & 1.13 & 4.06 & 2.66 & 2.90 & 0.155 & 2.26 & 8.00 & 0.30 & 0.09 & 5.70 \\
\hline $358-10-3,73-75$ & 61.00 & 1.04 & 14.90 & 0.86 & 6.52 & 0.05 & 1.13 & 3.25 & 3.06 & 1.90 & 0.192 & 1.63 & 5.74 & 0.12 & 0.07 & 4.24 \\
\hline $358-15-1,66-68$ & 56.70 & 1.01 & 17.44 & 0.29 & 7.64 & 0.06 & 0.85 & 3.55 & 4.05 & 1.60 & 0.210 & 0.00 & 6.40 & 0.05 & 0.09 & 6.44 \\
\hline $359-3-5,49-51$ & 23.01 & 0.97 & 6.63 & 0.86 & 3.09 & 0.04 & 31.73 & 1.22 & 1.37 & 2.90 & 0.384 & 25.28 & 26.70 & 0.10 & 0.10 & 1.66 \\
\hline $359-2-6,139-141$ & 10.05 & 0.41 & 2.33 & 0.36 & 2.67 & 0.03 & 42.24 & 1.22 & 0.71 & 2.01 & 0.711 & 35.26 & 36.82 & 0.23 & 0.09 & 1.41 \\
\hline
\end{tabular}

TABLE 4

Contents of Carbonate Minerals in Bottom Sediments From Leg 39 (in \%)

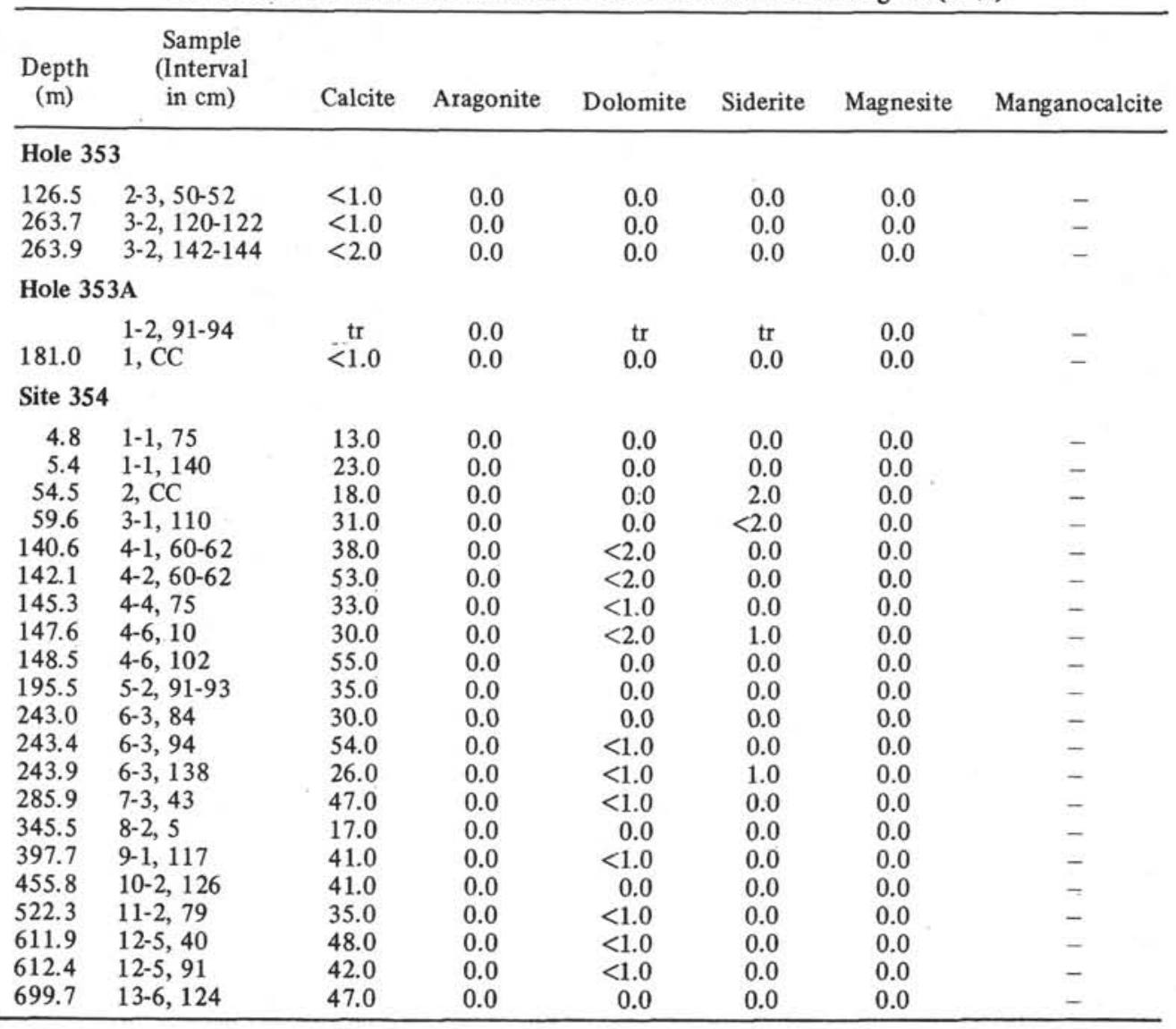


TABLE 4 - Continued

\begin{tabular}{|c|c|c|c|c|c|c|c|}
\hline $\begin{array}{l}\text { Depth } \\
\text { (m) }\end{array}$ & $\begin{array}{l}\text { Sample } \\
\text { (Interval } \\
\text { in } \mathrm{cm} \text { ) }\end{array}$ & Calcite & Aragonite & Dolomite & Siderite & Magnesite & Manganocalcite \\
\hline 705.4 & $14-4,35-40$ & 51.0 & 0.0 & 0.0 & 0.0 & 0.0 & - \\
\hline 820.9 & $15-2,35$ & 47.0 & 0.0 & 0.0 & 0.0 & 0.0 & - \\
\hline 823.4 & $15-3,138$ & 31.0 & 0.0 & 0.0 & 0.0 & 0.0 & - \\
\hline 835.5 & $16-2,47$ & 36.0 & 0.0 & 0.0 & 0.0 & 0.0 & - \\
\hline 842.2 & $16-6,124$ & 37.0 & 0.0 & 0.0 & 0.0 & 0.0 & - \\
\hline 858.3 & $17-1,127$ & 34.0 & 0.0 & 6.0 & 4.0 & 0.0 & - \\
\hline 872.8 & $18-1,130$ & 42.0 & 0.0 & $<1.0$ & 0.0 & 0.0 & - \\
\hline 877.3 & $18-4,127$ & 27.0 & 0.0 & 0.0 & 0.0 & 0.0 & - \\
\hline \multicolumn{8}{|c|}{ Site 355} \\
\hline 55.2 & $1-2,72-74$ & 0.0 & 0.0 & 0.0 & 0.0 & - & 0.0 \\
\hline 113.5 & $2-3,50-52$ & 0.0 & 0.0 & 0.0 & 0.0 & - & 0.0 \\
\hline 117.3 & $2-5,130-132$ & 0.0 & 0.0 & 0.0 & $\mathrm{tr}$ & - & $\operatorname{tr}$ \\
\hline 169.4 & $3-2,90-92$ & 33.0 & 0.0 & 0.0 & 0.0 & - & 0.0 \\
\hline 173.6 & $3-5,60-62$ & 0.0 & 0.0 & 0.0 & 0.0 & - & $\operatorname{tr}$ \\
\hline 223.1 & $4-3,110-112$ & 0.0 & 0.0 & 0.0 & 0.0 & - & $\mathrm{tr}$ \\
\hline 243.4 & $5-1,38-40$ & 0.0 & 0.0 & 0.0 & $<2.0$ & - & 3.0 \\
\hline 248.5 & $5-4,100-102$ & 0.0 & 0.0 & 0.0 & $<1.0$ & - & 2.0 \\
\hline 264.2 & $6-2,66-68$ & 0.0 & 0.0 & 0.0 & $<1.0$ & - & 1.0 \\
\hline 287.9 & $7-2,90-92$ & 0.0 & 0.0 & 0.0 & $<2.0$ & - & 3.0 \\
\hline 289.5 & $7-3,100-102$ & 0.0 & 0.0 & 0.0 & $<1.0$ & - & 2.0 \\
\hline 308.7 & $8-2,120-121$ & 0.0 & 0.0 & 0.0 & $<2.0$ & - & 4.0 \\
\hline 321.1 & $9-2,58-60$ & 0.0 & 0.0 & 0.0 & 0.0 & - & 1.0 \\
\hline 349.9 & $11-2,86-88$ & 0.0 & 0.0 & 0.0 & $\operatorname{tr}$ & - & 2.0 \\
\hline 363.9 & $12-5,86-88$ & 0.0 & 0.0 & 0.0 & 0.0 & - & tr \\
\hline 374.8 & $13-3,75-77$ & 0.0 & 0.0 & 0.0 & 0.0 & - & tr \\
\hline 386.4 & $15-1,86$ & 0.0 & 0.0 & $3.0(?)$ & 0.0 & - & tr \\
\hline 386.5 & $15-1,101$ & 0.0 & 0.0 & 0.0 & 0.0 & - & tr \\
\hline 408.9 & $17-3,142-145$ & 87.0 & 0.0 & 0.0 & 0.0 & - & 0.0 \\
\hline 409.2 & $17-4,20-22$ & 79.0 & 0.0 & 0.0 & 0.0 & - & 0.0 \\
\hline 412.7 & $17-6,65-67$ & 89.0 & 0.0 & 0.0 & 0.0 & - & 0.0 \\
\hline 419.7 & $18-1,120-122$ & 82.0 & 0.0 & 0.0 & 0.0 & - & 0.0 \\
\hline 421.9 & $18-3,41-43$ & 72.0 & 0.0 & 0.0 & 0.0 & - & 0.0 \\
\hline 430.8 & $19-2,131-133$ & 61.0 & 0.0 & 0.0 & 0.0 & - & 0.0 \\
\hline 442.0 & $20-2,148-150$ & 86.0 & 0.0 & 0.0 & 0.0 & - & 0.0 \\
\hline \multicolumn{8}{|c|}{ Hole 356} \\
\hline 10.3 & $2-1,82-85$ & 67.0 & 0.0 & 0.0 & 0.0 & - & 0.0 \\
\hline 39.8 & $3-2,28-30$ & 52.0 & 0.0 & 0.0 & 0.0 & - & 0.0 \\
\hline 60.6 & $4-3,55-57$ & 42.0 & 0.0 & 0.0 & 0.0 & - & 0.0 \\
\hline 91.0 & $5-4,101-103$ & 52.0 & 0.0 & 0.0 & 0.0 & - & 0.0 \\
\hline 119.0 & $6-4,50-53$ & 30.0 & 0.0 & 0.0 & 0.0 & - & 0.0 \\
\hline 138.0 & $7-4,50-53$ & 24.0 & 0.0 & 0.0 & 0.0 & - & 0.0 \\
\hline 169.6 & $8-2,60-63$ & 30.0 & 0.0 & 0.0 & 0.0 & - & 0.0 \\
\hline 198.5 & $9-2,98-100$ & 36.0 & 0.0 & 0.0 & tr & - & 0.0 \\
\hline 225.0 & $10-3,48-50$ & 29.0 & 0.0 & 0.0 & tr & - & 0.0 \\
\hline 244.8 & $11-2,129-131$ & 16.0 & 0.0 & 0.0 & 0.0 & - & 0.0 \\
\hline 253.1 & $12-1,11-13$ & 21.0 & 0.0 & 0.0 & 0.0 & - & 0.0 \\
\hline 273.8 & $14-1,25-27$ & 31.0 & 0.0 & 0.0 & $\operatorname{tr}$ & - & 0.0 \\
\hline 292.0 & $16-1,100-102$ & 17.0 & 0.0 & 0.0 & 0.0 & - & 0.0 \\
\hline 299.5 & $17-4,50-53$ & 44.0 & 0.0 & 0.0 & 0.0 & - & 0.0 \\
\hline 318.4 & $19-4,40-42$ & 16.0 & 0.0 & 0.0 & 0.0 & - & 0.0 \\
\hline 353.7 & $23-2,70-72$ & 38.0 & 0.0 & 0.0 & 0.0 & - & 0.0 \\
\hline 365.0 & $24-3,101-103$ & 21.0 & 0.0 & 0.0 & 0.0 & - & 0.0 \\
\hline 365.9 & $24-4,35-37$ & 70.0 & 0.0 & 0.0 & 0.0 & - & 0.0 \\
\hline 368.2 & $24-5,120-122$ & 42.0 & 0.0 & 0.0 & 0.0 & - & 0.0 \\
\hline 372.8 & $25-2,30-33$ & 50.0 & 0.0 & 0.0 & tr & - & 0.0 \\
\hline 381.7 & $26-2,23-25$ & 46.0 & 0.0 & 0.0 & tr & - & 0.0 \\
\hline 408.8 & $29-1,30-32$ & 8.0 & 0.0 & 0.0 & 0.0 & - & 0.0 \\
\hline $411.1^{\prime}$ & $29-2,110-112$ & 24.0 & 0.0 & 0.0 & 0.0 & - & 0.0 \\
\hline 416.7 & $29-6,30-32$ & 50.0 & 0.0 & 0.0 & 0.0 & - & 0.0 \\
\hline 443.7 & $31-5,68-70$ & 63.0 & 0.0 & 0.0 & 0.0 & - & 0.0 \\
\hline 487.0 & $33-2,94-97$ & 45.0 & 0.0 & 0.0 & 0.0 & - & 0.0 \\
\hline 545.0 & $35-3,50-52$ & 46.0 & 0.0 & 0.0 & 0.0 & - & 0.0 \\
\hline 654.3 & $38-3,82-84$ & 45.0 & 0.0 & tr & $\operatorname{tr}$ & - & 0.0 \\
\hline 681.3 & $39-5,75-77$ & 0.0 & 0.0 & 0.0 & 0.0 & - & 0.0 \\
\hline 701.4 & $40-6,42-44$ & 2.0 & 0.0 & 0.0 & 0.0 & - & 0.0 \\
\hline 705.0 & $41-2,48-50$ & 0.0 & 0.0 & 0.0 & 0.0 & - & 0.0 \\
\hline 734.1 & $44-2,110-112$ & 26.0 & 0.0 & 0.0 & 0.0 & - & 0.0 \\
\hline
\end{tabular}


TABLE 4 - Continued

\begin{tabular}{|c|c|c|c|c|c|c|c|}
\hline $\begin{array}{c}\text { Depth } \\
\text { (m) }\end{array}$ & $\begin{array}{l}\text { Sample } \\
\text { (Interval } \\
\text { in } \mathrm{cm} \text { ) }\end{array}$ & Calcite & Aragonite & Dolomite & Siderite & Magnesite & Manganocalcite \\
\hline \multicolumn{8}{|c|}{ Hole 356A } \\
\hline 24.2 & $1-4,72-74$ & 34.0 & 0.0 & 0.0 & 0.0 & - & 0.0 \\
\hline 35.1 & $2-5,55-57$ & 43.0 & 0.0 & 0.0 & 0.0 & - & 0.0 \\
\hline \multicolumn{8}{|c|}{ Hole 359} \\
\hline 6.4 & $1-3,91-141$ & 74.0 & 0.0 & 0.0 & 0.0 & - & 0.0 \\
\hline 36.9 & $2-6,139-141$ & 58.0 & 0.0 & 0.0 & 0.0 & - & 0.0 \\
\hline 58.2 & $3-1,15-17$ & 47.0 & 0.0 & 0.0 & 0.0 & - & 0.0 \\
\hline 62.0 & $3-3,96-98$ & 40.0 & 0.0 & 0.0 & 0.0 & - & 0.0 \\
\hline 62.8 & $3-4,26-28$ & 0.0 & 0.0 & 0.0 & $<1.0$ & - & 0.0 \\
\hline 91.2 & $4-2,19-21$ & 3.0 & 0.0 & 0.0 & 0.0 & - & 0.0 \\
\hline \multicolumn{8}{|c|}{ Hole 359A } \\
\hline 17.6 & $1-6,108-110$ & 73.0 & 0.0 & 0.0 & 0.0 & - & 0.0 \\
\hline 17.6 & $1-6,112-114$ & 72.0 & 0.0 & 0.0 & 0.0 & - & 0.0 \\
\hline 21.5 & $2-2,104-106$ & 72.0 & 0.0 & 0.0 & 0.0 & - & 0.0 \\
\hline
\end{tabular}

Unit IV is represented by slightly cemented nannofossil oozes (chalk) and limestones containing $\mathrm{CaCO}_{3}$ in the range of 44.03 to $87.05 \%$, with carbonate decreasing from top to bottom. In addition to coccoliths and foraminifers, dolomite is found locally (up to $10-15 \%$ of the sediment).

Unit $\mathrm{V}$ consists of marly muds and siliceous limestones; the content of $\mathrm{CaCO}_{3}$ is 40.27 to $67.29 \%$. The basic components are authigenic calcite, coccoliths, and planktonic foraminifers. At the very bottom of the unit a calcareous concretion of $10 \mathrm{~cm}$ was found. In the upper part of Unit V (Core 40, Section 4 and Core 41, Section 2) are found interlayers of terrigenous (siliceous) sediments, containing 1 to $15.01 \%$ of $\mathrm{CaCO}_{3}$, enriched by $\mathrm{Zn}, \mathrm{Ni}, \mathrm{Co}$, and very low in potassium.

Thus, the quantity of $\mathrm{CaCO}_{3}$ decreases from top to bottom. The role of foraminifers decreases in the same direction, and authigenic calcite and biogenic siliceous remains increase.

Site 357 presents a classical example of transition from loose foraminiferal-coccolith ooze of PlioPleistocene age into chalk and limestones of Cretaceous age.

\section{Site 358}

The content of $\mathrm{CaCO}_{3}$ varies in the range from 0.00 to $61.29 \%$. In Unit $\mathrm{I} \mathrm{CaCO}_{3}$ is completely absent. It is possibly related to: (1) unfavorable (cold) conditions for organisms with calcareous skeletons; (2) with position of the site below the present compensation depth (4600-5000 $\mathrm{m}$ for the Argentine Basin); or (3) with intrusion of cold Antarctic bottom waters into the basin.

The lower unit of sediments is represented by marly chalks and terrigenous muds (mudstones). The calcareous material consists mostly of coccoliths, with foraminifers occurring more rarely. This material consists exclusively of low-magnesian calcite (Table 5). The content of $\mathrm{CaCO}_{3}$ decreases to $1.75 \%$ in mudstones.

The high $\mathrm{CaCO}_{3}$ content indicates that the depth had been either close to the compensation depth or above it during the period of accumulation of the sediments of the lower unit. Large amounts of barite are in the chalk and Paleogene sediments of Site 358 (Figure 2).

After the deposition of Unit II sediments (late Eocene?), the conditions of sedimentation abruptly changed. This change represents not only a possible variation (rise) of the compensation depth (or subsidence of the bottom below it), but also a time of general drop of temperature in the southern hemisphere, beginning in the Eocene when local glaciers

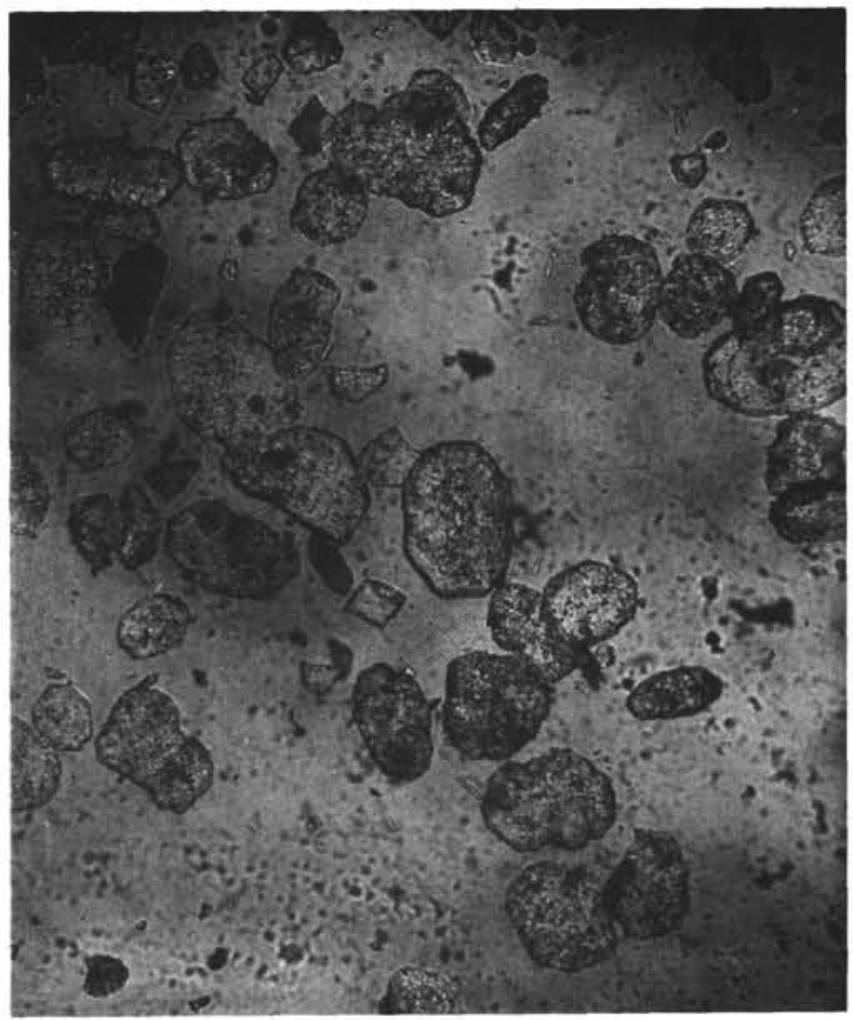

Figure 2. Microphotograph of barite at the Site 358 (Sample 11-4, 91-93 cm). Heavy coarse aleurite fraction (0.1$0.05 \mathrm{~mm}$ ). 
TABLE 5

X-ray Diffraction Analysis of Bulk Samples From Sites 357, 358, Leg 39 (in \% from total crystalline componnets)

\begin{tabular}{|c|c|c|c|c|c|c|}
\hline $\begin{array}{l}\text { Depth } \\
\text { (m) }\end{array}$ & $\begin{array}{c}\text { Sample } \\
\text { (Interval } \\
\text { in } \mathrm{cm} \text { ) }\end{array}$ & Calcite & Dolomite & Siderite & Total & Pyrite \\
\hline 4.0 & $1-3,100-103$ & 95.2 & 0.0 & 0.0 & 95.2 & 0.0 \\
\hline 20.4 & $3-2,90-92$ & 93.5 & 3.9 & 0.0 & 97.4 & 0.0 \\
\hline 41.0 & $5-3,100-102$ & 90.5 & 0.0 & 0.0 & 90.5 & 0.0 \\
\hline 51.8 & $6-4,80-83$ & 91.3 & 0.0 & 0.0 & 91.3 & 0.0 \\
\hline 67.7 & $8-2,70-73$ & 81.0 & 0.0 & 0.0 & 81.0 & 0.0 \\
\hline 77.2 & $9-2,70-73$ & 96.2 & 0.0 & 0.0 & 96.2 & 0.0 \\
\hline 88.3 & $10-1,80-82$ & 94.3 & 0.0 & 0.0 & 94.3 & 0.0 \\
\hline 89.8 & $10-2,80-82$ & 92.8 & 0.0 & 0.0 & 92.8 & 0.0 \\
\hline 112.1 & $11-2,103-106$ & 96.6 & 0.0 & 0.0 & 96.6 & 0.0 \\
\hline 137.1 & $13-4,62-65$ & 81.8 & 0.0 & 0.0 & 81.8 & 0.0 \\
\hline 178.3 & $15-2,78-81$ & 90.6 & 0.0 & 0.0 & 90.6 & 0.0 \\
\hline 193.0 & $17-3,100-102$ & 89.2 & 0.0 & 0.0 & 89.2 & 0.0 \\
\hline 196.0 & $17-5,100-102$ & 80.8 & 0.0 & 0.0 & 80.8 & 0.0 \\
\hline 262.7 & $20-2,117-120$ & 88.4 & 0.0 & 0.0 & 88.4 & 0.0 \\
\hline 309.2 & $22-4,13-16$ & 88.1 & 0.0 & 0.0 & 88.1 & 0.0 \\
\hline 333.6 & $23-2,61-64$ & 91.2 & 0.0 & 0.0 & 91.2 & 0.0 \\
\hline 351.0 & $24-1,45-48$ & 86.1 & 3.4 & 0.0 & 89.5 & 0.0 \\
\hline 357.4 & $24-5,84-87$ & 82.3 & 10.2 & 0.0 & 92.5 & 0.0 \\
\hline 359.0 & $24-6,101-104$ & 0.0 & 100.0 & 0.0 & 100.0 & 0.0 \\
\hline 359.3 & $24-6,132-135$ & 0.0 & 100.0 & 0.0 & 100.0 & 0.0 \\
\hline 383.5 & $26-3,148-151$ & 100.0 & 0.0 & 0.0 & 100.0 & 0.0 \\
\hline 411.6 & $27-3,108-111$ & 87.9 & 0.0 & 0.0 & 87.9 & 0.0 \\
\hline 436.9 & $28-1,91$ & 57.6 & 0.0 & 0.0 & 57.6 & 0.0 \\
\hline 471.5 & $29-1,98-101$ & 88.5 & 0.0 & 0.0 & 88.5 & 0.0 \\
\hline 474.3 & $30-1,31-34$ & 94.8 & 0.0 & 0.0 & 94.8 & 0.0 \\
\hline 498.7 & $31-2,123-126$ & 91.6 & 0.0 & 0.0 & 91.6 & 0.0 \\
\hline 525.9 & $33-3,142-145$ & 68.0 & 9.5 & 0.0 & 77.5 & 0.0 \\
\hline 554.8 & $84-4,32-35$ & 71.9 & $\mathrm{tr}$ & 0.0 & 71.9 & 0.0 \\
\hline 607.7 & $36-1,68-71$ & 52.8 & 7.0 & 0.0 & 59.8 & 0.0 \\
\hline 693.3 & $40-1,74-77$ & 61.0 & 15.4 & 0.0 & 76.4 & 0.0 \\
\hline 697.4 & $40-4,44-45$ & 17.4 & 0.0 & 0.0 & 17.4 & 0.0 \\
\hline 697.5 & $40-4,45-46$ & tr & 0.0 & 0.0 & $\operatorname{tr}$ & $\operatorname{tr}$ \\
\hline 705.3 & $41-2,76-79$ & 51.4 & tr & 0.0 & 51.4 & 0.0 \\
\hline 705.3 & $41-2,79-80$ & 17.9 & 0.0 & 0.0 & 17.9 & 6.3 \\
\hline 705.3 & $41-2,81-82$ & 19.5 & 0.0 & 0.0 & 19.5 & 8.6 \\
\hline 718.0 & $42-3,96-99$ & 49.7 & 11.1 & 2.0 & 62.8 & 0.0 \\
\hline 726.4 & $43-3,140-143$ & 50.3 & 1.8 & 1.4 & 53.6 & 0.0 \\
\hline 733.2 & $44-2,18-21$ & 67.2 & 10.3 & 0.0 & 77.5 & 0.0 \\
\hline 746.3 & $46-3,31-34$ & 77.8 & 0.0 & tr & 77.8 & 0.0 \\
\hline 753.1 & $47-3,56-59$ & 59.6 & 18.0 & 0.0 & 77.6 & 0.0 \\
\hline 759.5 & $48-1,47-48$ & 82.2 & 0.0 & 0.0 & 82.0 & 0.0 \\
\hline 781.4 & $50-3,42-44$ & 80.7 & 6.8 & 0.0 & 87.5 & 0.0 \\
\hline 790.9 & $51-3,34-36$ & 62.1 & 0.0 & 0.0 & 62.1 & 0.0 \\
\hline 796.2 & $51-6,125-126$ & 64.5 & 0.0 & 0.0 & 64.5 & 0.0 \\
\hline \multicolumn{7}{|c|}{ Site 358} \\
\hline 55.7 & $1-6,70-72$ & 0.0 & tr & 4.4 & 4.4 & 0.0 \\
\hline 128.9 & $2-4,94-96$ & 0.0 & $\operatorname{tr}$ & 4.3 & 4.3 & 0.0 \\
\hline 201.7 & $3-2,71-73$ & 0.0 & 0.0 & 0.0 & 0.0 & 0.0 \\
\hline 206.2 & $3-5,71-73$ & 0.0 & 0.0 & 0.0 & 0.0 & 0.0 \\
\hline 282.5 & $4-1,96-98$ & 0.0 & 0.0 & 3.6 & 3.6 & 0.0 \\
\hline 359.6 & $5-2,62-64$ & 0.0 & 0.0 & 3.1 & 3.1 & 0.0 \\
\hline 426.9 & $6-3,139-141$ & 0.0 & 0.0 & 2.9 & 2.9 & 0.0 \\
\hline 493.0 & $7-2,99-101$ & 0.0 & 0.0 & 0.0 & 0.0 & 0.0 \\
\hline 552.2 & $8-1,117-119$ & 0.0 & 0.0 & 0.0 & 0.0 & 0.0 \\
\hline 595.6 & $9-2,63-65$ & 0.0 & 0.0 & 4.0 & 4.0 & 0.0 \\
\hline 640.2 & $10-3,73-75$ & 0.0 & 0.0 & 0.0 & 0.0 & 0.0 \\
\hline 706.1 & $11-3,7-9$ & 0.0 & 0.0 & 0.0 & 0.0 & 0.0 \\
\hline 708.4 & $11-4,86-88$ & 86.7 & 0.0 & 0.0 & 86.7 & 0.0 \\
\hline 752.6 & $12-2,59-61$ & 64.3 & 0.0 & 0.0 & 64.3 & 0.0 \\
\hline 754.3 & $12-3,75-77$ & 89.1 & 0.0 & 0.0 & 89.1 & 0.0 \\
\hline 781.8 & $13-2,130-132$ & 86.6 & 0.0 & 0.0 & 86.6 & 0.0 \\
\hline 791.4 & $14-2,137-139$ & 70.8 & 0.0 & 0.0 & 70.8 & 0.0 \\
\hline 804.7 & $15-1,66-68$ & 8.9 & 0.0 & 0.0 & 3.9 & 0.0 \\
\hline 825.5 & $16-2,103-105$ & 22.6 & 0.0 & 0.0 & 22.6 & 0.0 \\
\hline
\end{tabular}


TABLE 6

Comparative Data on the Chemical Composition of the Recent and Upper Quaternary Sediments in Areas Near Leg 39 Sites (see Figure 1 for locations)

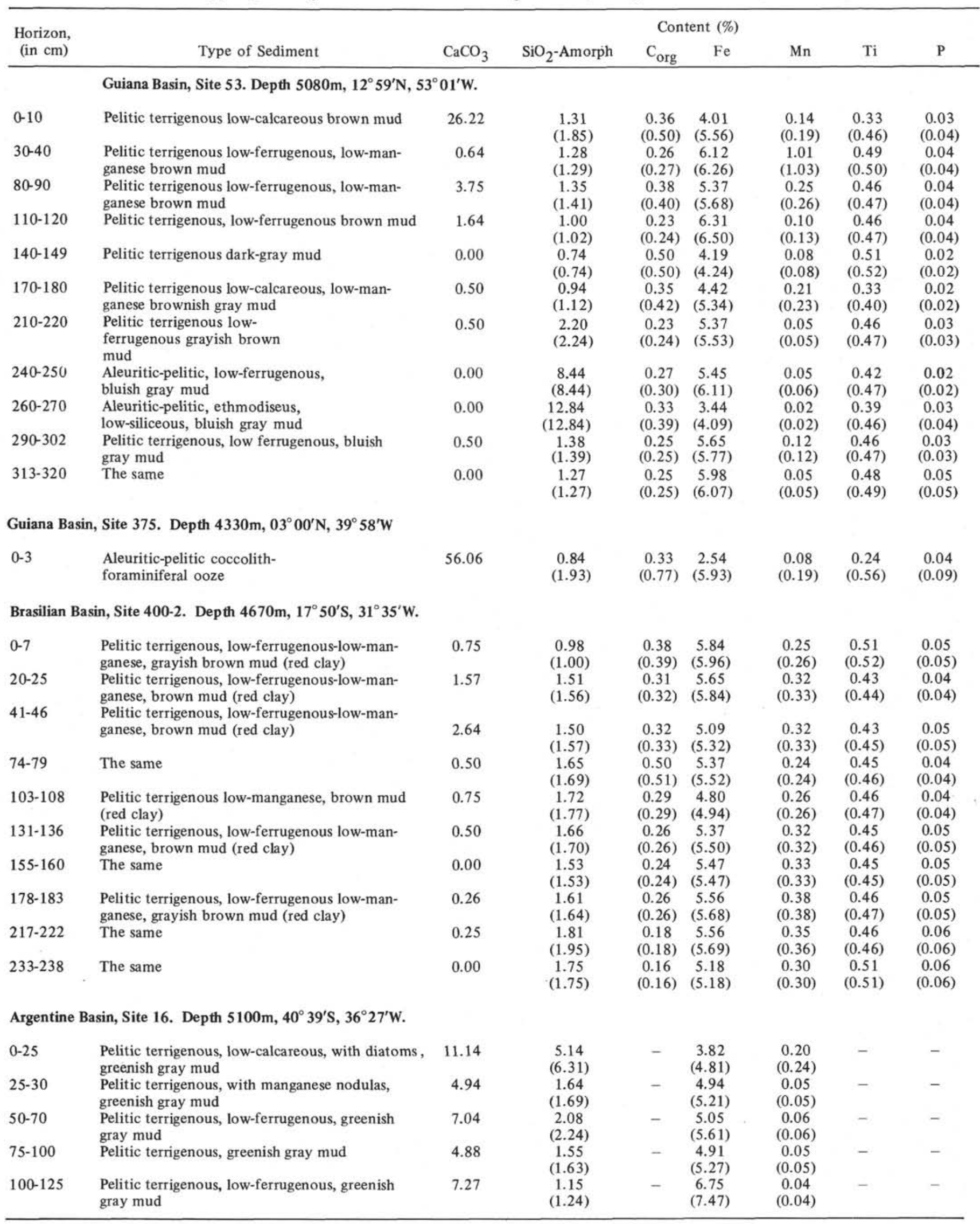


TABLE 6 - Continued

\begin{tabular}{|c|c|c|c|c|c|c|c|c|}
\hline \multirow{2}{*}{$\begin{array}{r}\text { Horizon } \\
\text { (in } \mathrm{cm} \text { ) }\end{array}$} & \multirow[b]{2}{*}{ Type of Sediment } & \multicolumn{6}{|c|}{ Content $(\%)$} & \multirow[b]{2}{*}{$\mathbf{P}$} \\
\hline & & $\mathrm{CaCO}_{3}$ & $\mathrm{SiO}_{2}$-Amorph & $\mathrm{C}_{\text {org }}$ & $\mathrm{Fe}$ & Mn & $\mathrm{Ti}$ & \\
\hline \multicolumn{9}{|c|}{ Argentine Basin - Continued } \\
\hline $125-150$ & Pelitic terrigenous, greenish gray mud & 6.59 & $\begin{array}{c}1.13 \\
(1.21)\end{array}$ & - & $\begin{array}{l}4.88 \\
(5.36)\end{array}$ & $\begin{array}{l}0.05 \\
(0.05)\end{array}$ & - & - \\
\hline $150-175$ & $\begin{array}{l}\text { Pelitic terrigenous, low-ferrugenous, greenish } \\
\text { gray mud }\end{array}$ & 4.32 & $\begin{array}{l}1.12 \\
(1.17)\end{array}$ & - & $\begin{array}{r}5.22 \\
(6.43\end{array}$ & $\begin{array}{l}0.05 \\
(0.05)\end{array}$ & - & - \\
\hline $175-200$ & $\begin{array}{l}\text { Pelitic terrigenous, low- ferrugenous, greenish } \\
\text { gray mud }\end{array}$ & 9.66 & $\begin{array}{c}1.32 \\
(1.46)\end{array}$ & - & $\begin{array}{l}5.64 \\
(6.43)\end{array}$ & $\begin{array}{l}0.07 \\
(0.08)\end{array}$ & - & - \\
\hline $200-225$ & $\begin{array}{l}\text { Pelitic terrigenous, low-ferrugenous, low-cal- } \\
\text { careous, light-green mud }\end{array}$ & 16.94 & $\begin{array}{l}1.08 \\
(1.30)\end{array}$ & - & $\begin{array}{l}5.66 \\
(6.98)\end{array}$ & $\begin{array}{l}0.05 \\
(0.06)\end{array}$ & - & - \\
\hline $225-250$ & The same & 14.34 & 1.21 & - & 5.71 & 0.04 & - & - \\
\hline
\end{tabular}

Content of elements in dry sediment and on a carbonate-free basis (in brackets).

TABLE 7

Average Contents of Elements in Extended Genetic Types of Sediments in Sites 353 to 358 (see Table 2)

\begin{tabular}{|c|c|c|c|c|c|c|c|c|c|c|c|c|c|c|c|c|}
\hline \multirow[b]{2}{*}{ Type of Sediment } & \multirow[b]{2}{*}{ Sites } & \multirow[b]{2}{*}{$\begin{array}{c}\text { Total } \\
\text { Samples }\end{array}$} & \multicolumn{8}{|c|}{ Content (in \%) } & \multicolumn{6}{|c|}{ Content in $10^{-4 \%}$} \\
\hline & & & $\mathrm{CaCO}_{3}$ & $\mathrm{C}_{\text {org }}$ & $\mathrm{Fe}$ & $\mathrm{Mn}$ & $\mathrm{Ti}$ & $P$ & $\mathrm{Na}_{2} \mathrm{O}$ & $\mathrm{K}_{2} \mathrm{O}$ & $\mathrm{Zn}$ & $\mathrm{Cu}$ & $\mathrm{Ni}$ & Co & $\mathrm{Cr}_{\mathrm{r}}$ & $\mathrm{Cd}$ \\
\hline \multirow{2}{*}{$\begin{array}{l}\text { Terrigenous } \\
\text { sediments } \\
\left(<30 \% \mathrm{CaCO}_{3}\right)\end{array}$} & $\begin{array}{l}353^{\mathrm{a}}, 354^{\mathrm{a}}, 356^{\mathrm{a}}, \\
357^{\mathrm{a}}, 358^{\mathrm{a}}\end{array}$ & 13 & 9.43 & 0.46 & 4.55 & 0.11 & 0.50 & 0.06 & 2.09 & 2.30 & 120 & 45 & 84 & 26 & 49 & $<5$ \\
\hline & $\begin{array}{l}353^{\mathrm{b}}, 354^{\mathrm{b}}, 356^{\mathrm{b}} \\
357^{\mathrm{b}}, 358^{\mathrm{b}}\end{array}$ & 13 & - & - & 5.04 & 0.13 & 0.56 & 0.06 & 2.29 & 2.54 & 132 & 50 & 91 & 28 & 54 & $<6$ \\
\hline \multirow{5}{*}{$\begin{array}{l}\text { Siliceous muds } \\
\left(<30 \% \mathrm{CaCO}_{3}\right)\end{array}$} & $356^{\mathrm{a}}$ & 9 & 28.79 & 0.68 & 2.76 & 0.09 & 0.30 & - & 1.81 & 1.46 & 72 & 28 & 18 & 10 & 47 & $<6$ \\
\hline & $356^{\mathrm{b}}$ & 9 & - & - & 3.92 & 0.13 & 0.42 & - & 2.53 & 2.05 & 101 & 40 & 25 & 10 & 63 & $<9$ \\
\hline & $358^{\mathrm{a}, \mathrm{b}}$ & 9 & 0.00 & 0.28 & 4.46 & 0.06 & 0.49 & 0.04 & 2.6 & 2.57 & 118 & 74 & 47 & 18 & 38 & $<6$ \\
\hline & $356+358^{\mathrm{a}}$ & 18 & 28.79 & 0.48 & 3.61 & 0.07 & 0.39 & - & 3.03 & 2.43 & 95 & 52 & 33 & - & 42 & $<6$ \\
\hline & $356+358^{a, b}$ & 18 & - & - & 4.19 & 0.09 & 0.45 & -. & - & - & 110 & 57 & 36 & - & 50 & $<7$ \\
\hline \multirow{6}{*}{$\begin{array}{l}\text { Calcareous } \\
\text { sediments } \\
\left(>60 \% \mathrm{CaCO}_{3}\right)\end{array}$} & $354^{\mathrm{a}}$ to $15-3^{\mathrm{c}}$ & 9 & 68.98 & 0.11 & 1.71 & 0.08 & 0.11 & 0.03 & 0.81 & 0.71 & 37 & 61 & 22 & - & 31 & $<4$ \\
\hline & $354^{\mathrm{a}, \mathrm{b}}$ to $15-3^{\mathrm{c}}$ & 9 & - & - & 5.97 & 0.25 & 0.40 & 0.09 & - & - & 130 & 207 & 78 & - & 104 & $<14$ \\
\hline & $357^{\mathrm{a}}$ to $28-\mathrm{I}^{\mathrm{c}}$ & 21 & 82.68 & 0.48 & 0.98 & 0.03 & 0.12 & - & 0.98 & 0.58 & 18 & 16 & 15 & - & 28 & $<6$ \\
\hline & $357^{\mathrm{b}}$ to $28-1^{\mathrm{c}}$ & 21 & - & - & 6.35 & 0.27 & 0.61 & - & - & - & 136 & 105 & 87 & - & 154 & $<46$ \\
\hline & $\begin{array}{l}354,355^{\mathrm{a}}, 356^{\mathrm{a}}, \\
357^{\mathrm{a}}, 358^{\mathrm{a}}\end{array}$ & 45 & 76.94 & 0.41 & 1.27 & 0.09 & 0.12 & 0.03 & 0.91 & 0.73 & 27 & 27 & 19 & - & 26 & $<6$ \\
\hline & $\begin{array}{l}354^{\mathrm{b}}, 355^{\mathrm{b}}, 356^{\mathrm{b}} \\
357^{\mathrm{b}}, 358^{\mathrm{b}}\end{array}$ & 45 & - & - & 6.04 & 0.40 & 0.52 & 0.12 & - & - & 132 & 119 & 90 & - & 122 & $<33$ \\
\hline $\begin{array}{l}\text { Marly carbona- } \\
\text { ceous sediments }\end{array}$ & $\begin{array}{l}354^{\mathrm{a}}, 356^{\mathrm{a}}, 357^{\mathrm{a}} \\
354^{\mathrm{a}}, \mathrm{b} \\
356^{\mathrm{a}}, \mathrm{b}\end{array}$ & b $\begin{array}{r}35 \\
35\end{array}$ & 49.32 & 0.77 & 2.66 & 0.07 & 0.34 & 0.04 & 1.14 & 1.58 & 66 & 42 & 46 & - & 60 & $<5$ \\
\hline $\begin{array}{l}\left.\text { (30-60\% } \mathrm{CaCO}_{3}\right) \\
\left(3 \mathrm{C}^{2}\right)\end{array}$ & $354^{\mathrm{a}, \mathrm{b}}, 356^{\mathrm{a}, \mathrm{b}}, 357^{\mathrm{a}, \mathrm{b}}$ & 35 & 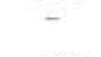 & - & 5.16 & 0.14 & 0.54 & 0.07 & 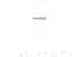 & - & 126 & 80 & 89 & - & 114 & $<11$ \\
\hline Pelagic muds & $355^{\mathrm{a}}$ & 21 & 1.27 & 0.39 & 5.28 & 0.18 & 0.57 & 0.05 & 1.84 & 2.30 & 132 & 62 & 76 & 25 & 67 & $<6$ \\
\hline (ancient clays) & & 21 & ․․ & - & 5.34 & 0.18 & 0.58 & 0.05 & - & - & 133 & 66 & 77 & 25 & 67 & $<6$ \\
\hline
\end{tabular}

${ }^{\mathrm{a}}$ Natural (dry) sediment.

${ }^{\mathrm{b}}$ Evaluated in carbonate free basis.

${ }^{c}$ From surface to noted depth.

began appearing in the Antarctic (Geitzenauer et al., 1968). More intensive glaciation occurred in the Oligocene and an ice surface, similar to that of the present, was formed in the Miocene. The fall of temperature in the Antarctic resulted in formation of cold water masses, which as near-bottom currents (Neumann and Pierson, 1966; Bulatov, 1971; Ewing et al., 1971) penetrated far to the north and probably crossed the Equator. This resulted in (1) the rise of the compensation depth level: (2) a sharp increase of cold Antarctic water productivity and mass development of phytoplankton with siliceous skeletons, leading to the accumulation of siliceous muds in the Argentine Basin.

Oligocene-Pleistocene sedimentation took place mainly in a reducing environment, evidenced by gray sediment color and the presence of pyrite and siderite (Table 5). In late Quaternary time, the climatic condition of the Argentine Basin had evidently become more moderate (warm), as a result of which the content of $\mathrm{CaCO}_{3}$ increases locally to $16.94 \%$, whereas $\mathrm{SiO}_{2}$ amorph drops to $1.08 \%$ (Table 6).

\section{Site 359}

The upper sediment unit (Core 2, Section 6 and Core 1 , Section 3) consists of foraminiferal-coccolithic ooze, the content of $\mathrm{CaCO}_{3}$ being respectively $71.8 \%$ and $89.31 \%$. Considerable authigenic magnesian calcite (5-6 mol. \% of $\mathrm{MgCO}_{3}$ ) occurs.

Unit II (from Core 4, Section 2 up to Core 3, Section 4) consists of foraminiferal (Core 3, Section 5), terrigenous (Core 3, Section 4), and volcanogenic (Core 4 , Section 2) sediments with the content of $\mathrm{CaCO}_{3}$ being low (up to $3.25 \%$ ). Authigenic magnesian calcite and dolomite occur in negligible quantities there.

\section{Organic Carbon}

The content of $\mathrm{C}_{\text {org }}$ in the sediments of the western part of the central Atlantic varies from 0.03 up to $9.81 \%$ 
TABLE 8

Average Contents of Elements in the Recent Sediments ( $0.5 \mathrm{~cm}$ layer) of the Atlantic Ocean (Emelyanov, Shurko, 1973, Emelyanov, 1974a, b, 1975; Emelyanov et al., 1975)

\begin{tabular}{|c|c|c|c|c|c|c|c|c|c|c|}
\hline \multirow[b]{2}{*}{$\begin{array}{l}\text { Type of } \\
\text { Sediments }\end{array}$} & \multicolumn{3}{|r|}{$\mathrm{C}_{\text {org }}$} & \multirow[b]{2}{*}{ Average } & \multicolumn{3}{|c|}{$\mathrm{Fe}$} & \multicolumn{3}{|c|}{$\mathrm{Mn}$} \\
\hline & & $\begin{array}{l}\text { uantity } \\
\text { imples }\end{array}$ & $\begin{array}{l}\text { Limits of } \\
\text { Contents }\end{array}$ & & $\begin{array}{l}\text { Quantity } \\
\text { Samples }\end{array}$ & $\begin{array}{l}\text { Limits of } \\
\text { Contents }\end{array}$ & Average & $\begin{array}{l}\text { Quantity } \\
\text { Samples }\end{array}$ & $\begin{array}{l}\text { Limits of } \\
\text { Contents }\end{array}$ & Average \\
\hline $\begin{array}{l}\text { Terrigenous sedi- } \\
\text { ments }\left(<10 \% \mathrm{CaCO}_{3}\right)\end{array}$ & $\begin{array}{l}\mathrm{a} \\
\mathrm{b}\end{array}$ & $\begin{array}{l}169 \\
168\end{array}$ & $\begin{array}{l}0.04-8.10 \\
0.04-8.90\end{array}$ & $\begin{array}{l}0.92 \\
0.98\end{array}$ & $\begin{array}{l}284 \\
270\end{array}$ & $\begin{array}{l}\text { tr.- } 9.97 \\
\text { tr.- }-7.35\end{array}$ & $\begin{array}{l}3.18 \\
3.22\end{array}$ & $\begin{array}{l}13.1 \\
128\end{array}$ & $\begin{array}{l}\operatorname{tr}-1.88 \\
\text { tr. }-2.00\end{array}$ & $\begin{array}{l}0.09 \\
0.10\end{array}$ \\
\hline $\begin{array}{l}\text { (and terrigenous } \\
\text { pelitic muds) }\end{array}$ & $\begin{array}{l}\mathrm{a} \\
\mathrm{b}\end{array}$ & $\begin{array}{l}53 \\
52\end{array}$ & $\begin{array}{l}0.10-12.20 \\
0.15-20.62\end{array}$ & $\begin{array}{l}0.84 \\
1.42\end{array}$ & $\begin{array}{l}55 \\
54\end{array}$ & $\begin{array}{l}0.74-7.00 \\
0.80-7.35\end{array}$ & $\begin{array}{l}5.01 \\
5.34\end{array}$ & $\begin{array}{l}28 \\
28\end{array}$ & $\begin{array}{l}0.02-1.88 \\
0.03-2.00\end{array}$ & $\begin{array}{l}0.19 \\
0.20\end{array}$ \\
\hline $\begin{array}{l}\text { Mixed biogenic-terrig. } \\
\left(30-50 \% \mathrm{CaCO}_{3}\right)\end{array}$ & $\begin{array}{l}\mathrm{a} \\
\mathrm{b}\end{array}$ & $\begin{array}{l}18 \\
18\end{array}$ & $\begin{array}{l}0.37-8.10 \\
0.41-8.90\end{array}$ & $\begin{array}{l}1.84 \\
1.94\end{array}$ & $\begin{array}{l}123 \\
120\end{array}$ & $\begin{array}{l}0.75-6.71 \\
0.92-10.40\end{array}$ & $\begin{array}{l}2.96 \\
5.16\end{array}$ & $\begin{array}{l}64 \\
50\end{array}$ & $\begin{array}{l}\text { tr. }-0.39 \\
\text { tr. }-0.74\end{array}$ & $\begin{array}{l}0.10 \\
0.20\end{array}$ \\
\hline $\begin{array}{l}\text { Foram-sands nanno- } \\
\text { foram oozes } \\
\left(<50 \% \mathrm{CaCO}_{3}\right)\end{array}$ & $\begin{array}{l}\mathrm{a} \\
\mathrm{b}\end{array}$ & $\begin{array}{l}71 \\
63 .\end{array}$ & $\begin{array}{l}0.17-6.57 \\
0.38-20.08\end{array}$ & $\begin{array}{l}0.93 \\
3.60\end{array}$ & $\begin{array}{l}265 \\
263\end{array}$ & $\begin{array}{l}0.06-4.94 \\
0.50-16.24\end{array}$ & $\begin{array}{l}1.46 \\
5.71\end{array}$ & $\begin{array}{l}135 \\
110\end{array}$ & $\begin{array}{l}\operatorname{tr}--1.12 \\
\text { tr. }-2.98\end{array}$ & $\begin{array}{l}0.08 \\
0.29\end{array}$ \\
\hline $\begin{array}{l}\text { Diatom ooze } \\
\left(>30 \% \mathrm{SiO}_{2}\right) \text { amorph. }\end{array}$ & $\begin{array}{l}\mathrm{a} \\
\mathrm{b}\end{array}$ & 4 & $0.18-0.60$ & 0.34 & $\begin{array}{l}5 \\
5\end{array}$ & $\begin{array}{l}0.58-1.62 \\
1.58-4.14\end{array}$ & $\begin{array}{l}0.97 \\
2.85\end{array}$ & $\begin{array}{l}2 \\
2\end{array}$ & $\begin{array}{l}0.06-0.61 \\
0.14-1.67\end{array}$ & $\begin{array}{l}0.33 \\
0.90\end{array}$ \\
\hline $\begin{array}{l}\text { Volcanogenic } \\
\text { sediments } \\
\text { Iceland area }\end{array}$ & $\begin{array}{l}\mathrm{a} \\
\mathrm{b}\end{array}$ & $\begin{array}{l}31 \\
31\end{array}$ & $\begin{array}{l}0.12-1.90 \\
0.12-2.54\end{array}$ & $\begin{array}{l}0.92 \\
1.09\end{array}$ & $\begin{array}{l}66 \\
66\end{array}$ & $\begin{array}{l}5.04-11.85 \\
5.47-12.88\end{array}$ & $\begin{array}{l}7.07 \\
8.23\end{array}$ & $\begin{array}{l}36 \\
30\end{array}$ & $\begin{array}{l}0.05-0.24 \\
0.06-0.36\end{array}$ & $\begin{array}{l}0.15 \\
0.17\end{array}$ \\
\hline $\begin{array}{l}\text { Red clays } \\
\left(<10 \% \mathrm{CaCO}_{3}\right)\end{array}$ & $\begin{array}{l}\mathrm{a} \\
\mathrm{b}\end{array}$ & $\begin{array}{l}14 \\
14\end{array}$ & $\begin{array}{l}0.11-0.44 \\
0.11-0.45\end{array}$ & $\begin{array}{l}0.32 \\
0.33\end{array}$ & $\begin{array}{r}8 \\
14\end{array}$ & $\begin{array}{l}5.28-6.59 \\
4.92-13.52\end{array}$ & $\begin{array}{l}5.85 \\
6.43\end{array}$ & $\begin{array}{l}18 \\
14\end{array}$ & $\begin{array}{l}0.08-1.15 \\
0.23-3.62\end{array}$ & $\begin{array}{l}0.40 \\
0.71\end{array}$ \\
\hline $\begin{array}{l}\text { All types of } \\
\text { sediments }\end{array}$ & $\begin{array}{l}\mathrm{a} \\
\mathrm{b}\end{array}$ & $\begin{array}{l}452 \\
435\end{array}$ & $\begin{array}{l}0.04-12.50 \\
0.04-20.62\end{array}$ & $\begin{array}{l}1.09 \\
1.67\end{array}$ & $\begin{array}{l}959 \\
947\end{array}$ & $\begin{array}{l}\text { tr.-16.05 } \\
\text { tr.-19.94 }\end{array}$ & $\begin{array}{l}3.05 \\
4.88\end{array}$ & $\begin{array}{l}494 \\
428\end{array}$ & $\begin{array}{l}\text { tr.- }-3.14 \\
\text { tr.- }-3.62\end{array}$ & $\begin{array}{l}0.10 \\
0.19\end{array}$ \\
\hline
\end{tabular}

or 0.4 to $0.5 \%$ on the average. It is lower than in the Recent sediments of the Atlantic Ocean, but approximately the same as reported from Cenozoic sediments at sites drilled on Legs 3 and 4 (Pimm, A.C., 1970; Pimm, A.C., 1970).

The lowest contents of $\mathrm{C}_{\text {org }}$ (from $0.03-0.27 \%$ ) occur in biogenic calcareous sediments at Site 354. They are slightly lower than in pelagic terrigenous muds of late Quaternary age in the Guiana Basin (Table 2). This is a result of low biological productivity of this area in the Late Cretaceous and Cenozoic, and of low rates of sedimentation and rapid oxidation of organic material. In the Miocene the sedimentation rate was as low as $0.004 \mathrm{~cm} / 1000$ years (probably representing important hiatuses). In the Eocene-Oligocene-Miocene the rate was $1.7-2.2 \mathrm{~cm} / 1000$ years, compared to $10 \mathrm{~cm} / 1000$ years in the Pleistocene.

The content of $\mathrm{C}_{\text {org }}$ at Site 355 varies in the range of 0.15 to $0.66 \%$; i.e., it is very close to the average content in Recent red clays (Table 8). Approximately the same quantities of $\mathrm{C}_{\text {org }}$ occur in the pelagic muds at Site 358 . Thus, despite the high productivity of phytoplankton in Antarctic waters and waters of the Argentine Basin in the Oligocene-Pleistocene, the intensive accumulation of the remains of diatoms there, the rapid rates of sedimentation and, presumably, reducing environments of sedimentation, as little organic substance occurs there as in pelagic diatom muds from near Antarctica. Probably this is a result of rapid rates of decomposition of organic substances.

The $\mathrm{C}_{\text {org }}$ in the sediments of the Rio Grande Rise (Site 357 ) contain from 0.24 to $0.84 \%$, with an average of $0.50 \%$, which is approximately the same as in Recent coccolith-foraminiferal oozes of the west Atlantic (Emelyanov, 1975).

Abnormally high quantities of $\mathrm{C}_{\text {org }}$ (from $0.48-9.81 \%$ ) occur in older sediments at Site 356 , being 0.80 to $1.0 \%$ on the average. Interlayers of sediments with sapropels and pyrite accumulated there during Coniacian and Turonian time. Similar interlayers with sapropels were described in holes drilled during Leg 14 (Berger and von Rad, 1972), and also in the Caribbean Sea. The high quantities of organic substance are thought to have accumulated in situ under reducing conditions. High contents of $\mathrm{C}_{\text {org }}$ are independent of genetic type of sediment and depth of subbottom. The high contents of $\mathrm{C}_{\text {org }}$ are caused mainly by high constant biologic productivity, favorable conditions for burial and possible supply of organic material from outside the basin. The area of the ocean now occupied by the São Paulo Plateau was a semi-isolated (shallow) basin, supplied by terrigenous material from South America during the Late Cretaceous and Early Cenozoic. Rates of sedimentation were usually high $(2-6 \mathrm{~cm} / 1000$ years), and reducing conditions (pyrite is frequently present in sediments) were prevalent. Redeposition of sediment occurred, with the formation of conglomerates and turbidites.

\section{DISCUSSION AND CONCLUSIONS}

Tables 1 to 5, 7, and 9 show the relative abundance of a series of selected elements determined to be in cores of sediments from DSDP Leg 39 . Tables 6,8 , and 10 show their comparative abundance in a variety of ancient and modern marine lithofacies elsewhere in the Atlantic Ocean. Examination of this data shows that, in general, sediments of any one genetic type have a chemical composition that varies only slightly through space and time which suggests, in turn, that there has been essentially no geochemical "evolution" in the central Atlantic from Late Cretaceous to the present. In contrast, the geographic distribution of the lithofacies in that area has changed markedly during the same time 
TABLE 8 - Continued

\begin{tabular}{|c|c|c|c|c|c|c|c|c|c|c|c|}
\hline \multirow[b]{2}{*}{$\begin{array}{l}\text { Quantity } \\
\text { Samples }\end{array}$} & \multirow{2}{*}{$\begin{array}{c}\mathrm{Ti} \\
\text { Limits of } \\
\text { Contents }\end{array}$} & \multirow[b]{2}{*}{ Average } & \multicolumn{3}{|c|}{ P } & \multicolumn{3}{|c|}{$\mathrm{Ba} \cdot 10^{-4}$} & \multicolumn{3}{|c|}{$\mathrm{Zr} \cdot 10^{-4}$} \\
\hline & & & $\begin{array}{l}\text { Quantity } \\
\text { Samples }\end{array}$ & $\begin{array}{l}\text { Limits of } \\
\text { Contents }\end{array}$ & Average & $\begin{array}{l}\text { Quantity } \\
\text { Samples }\end{array}$ & $\begin{array}{l}\text { Limits of } \\
\text { Contents }\end{array}$ & Average & $\begin{array}{l}\text { Quantity } \\
\text { Samples }\end{array}$ & $\begin{array}{l}\text { Limits of } \\
\text { Contents }\end{array}$ & Average \\
\hline 188 & tr. -0.82 & 0.34 & 177 & tr. -0.46 & 0.07 & 100 & $130-3750$ & 470 & 103 & $300-850$ & 210 \\
\hline 177 & tr. -0.85 & 0.38 & 177 & tr. -0.48 & 0.08 & 100 & $130-4260$ & 500 & 103 & $300-860$ & 220 \\
\hline 53 & $0.04-0.66$ & 0.40 & 47 & tr. -0.46 & 0.08 & 22 & $130-3750$ & 690 & 22 & $80-270$ & 150 \\
\hline 46 & $0.11-0.71$ & 0.47 & 47 & $0.02-0.48$ & 0.08 & 22 & $130-4260$ & 740 & 22 & $90-290$ & 160 \\
\hline 94 & $0.05-0.81$ & 0.27 & 81 & $0.01-0.70$ & 0.09 & 42 & $100-1200$ & 450 & 42 & $<50-500$ & 150 \\
\hline 93 & $0.08-1.49$ & 0.47 & 81 & $0.02-1.16$ & 0.14 & 42 & $160-2100$ & 740 & 42 & $<70-1180$ & 240 \\
\hline \multirow{3}{*}{$\begin{array}{l}206 \\
204\end{array}$} & tr. -0.44 & 0.12 & 184 & tr.-4.36 & 0.11 & 80 & $<200-1830$ & 350 & 80 & $<20-670$ & 90 \\
\hline & tr. -1.30 & 0.48 & 183 & $0.01-9.13$ & 0.42 & 80 & $<200-7870$ & 460 & 80 & $<40-2220$ & 340 \\
\hline & & & & & & & & & & & \\
\hline 6 & $0.07-0.10$ & 0.08 & 6 & $0.02-0.03$ & 0.02 & 8 & $210-1040$ & 570 & 7 & $40-130$ & 70 \\
\hline 5 & $0.11-0.31$ & 0.21 & 5 & $0.03-0.06$ & 0.05 & 8 & $220-1090$ & 590 & - & - & - \\
\hline 36 & $0.52-1.96$ & 1.13 & 31 & $0.06-0.18$ & 0.11 & 29 & $<200-1300$ & 410 & 27 & $<40-360$ & 180 \\
\hline 32 & $0.70-2.36$ & 1.35 & 27 & $0.07-0.20$ & 0.13 & 29 & $<200-1870$ & 460 & 27 & $<40-370$ & 190 \\
\hline 15 & $0.11-0.66$ & 0.44 & 14 & $0.05-0.11$ & 0.08 & - & - & - & - & - . & - \\
\hline 10 & $0.41-0.57$ & 0.50 & 14 & $0.05-0.12$ & 0.08 & - & - & - & - & - & - \\
\hline 700 & tr.-1.96 & 0.31 & 623 & tr. -4.36 & 0.10 & 352 & $90-7870$ & 450 & 354 & $<20-850$ & 160 \\
\hline 682 & tr.- -2.36 & 0.50 & 615 & tr.- -9.13 & 0.21 & 352 & $110-7870$ & 780 & 354 & $<40-1630$ & 240 \\
\hline
\end{tabular}

interval, influenced by paleoenvironmental conditions and diagenesis.

$\mathrm{CaCO}_{3}$ data indicate that the conditions of carbonate accumulation changed sharply near the PaleoceneEocene boundary. This is particularly true of areas that were deep or distant from the continent of South America (i.e., Sites 355,357 , and 358).

In the Argentine Basin terrigenous sediment had been primarily accumulating in the Late Cretaceous; carbonates were meager. The conditions of sedimentation in Cretaceous time suggest the site was shallower than at present. During Paleocene and the beginning of Eocene time, biogenic calcareous material accumulated in greater quantities, producing chalk. Conditions then changed sharply with the onset of glaciation in the Antarctic and the generation of cold, near-bottom current, which penetrated into the Argentine Basin. The compensation depth became shallower, leading to dissolution of carbonates and accumulation of siliceous and silico-terrigenous sediments. This sedimentation regime lasted into the Pleistocene. In late PleistoceneHolocene time conditions appear to have moderated, favoring biogenic calcareous material as witnessed by an increase of $\mathrm{CaCO}_{3}$, up to $16 \%$ in Holocene sediments.

At the Rio Grande Rise site, in the Cretaceous, conditions for accumulation of $\mathrm{CaCO}_{3}$ were variable, perhaps because of the changing distance of the site from the continent and variations in rates of supply of terrigenous material. The depth of the Rio Grande Rise site itself may also have been changing. From late Maestrichtian to the present, the conditions of accumulation of $\mathrm{CaCO}_{3}$ were rather stable and favorable, resulting in a thick section (about $550 \mathrm{~m}$ ) of foraminiferal-nannofossil oozes. These oozes (beginning in the Miocene, at least) underwent diagenetic transformation (consolidation, cementation) and, down section, were converted into chalk and limestones. In addition, in the middle Eocene, dolomite occurs with limestones and marls.

Conditions for accumulation of biogenic carbonates in the Brazil Basin, during Late Cretaceous, were even more favorable than those in the Argentine Basin or on the Rio Grande Rise. The consistently high content of $\mathrm{CaCO}_{3}$ in the form of pelagic nannofossil oozes and chalk give evidence to this. In the Eocene an abrupt change occurred and conditions became unfavorable for $\mathrm{CaCO}_{3}$ accumulation. This was caused by either subsidence of the bottom, and/or intrusion of cold bottom waters from the Antarctic into the basin with consequent shoaling of the compensation depth. These conditions have remained essentially unchanged to the present and resulted in accumulation of noncarbonate pelagic muds and terrigenous sediments. The rates of sedimentation during this interval has been low, permitting extensive formation of zeolites.

In cases where sites were situated close to the continent (i.e., Sites 354,350 ) and where depths were moderate (above compensation), terrigenous material diluted the biogenic carbonate. At certain times (Late Cretaceous especially) the accumulation of carbonates was affected not only by dilution by terrigenous matter, but also by partial dissolution of carbonate, resulting from either subsidence of the bottom below compensation level or a rise in the compensation level itself.

The paucity of volcanogenic (pyroclastic) material is noteworthy (except at Site 359). In the Cretaceous period volcanic activity is evidenced but became much less active in Tertiary time; in only a few cases are there pyroclastics in the post-Cretaceous sediments. An example is the middle Eocene at Site 357 (Core 24, 
TABLE 8 - Continued

\begin{tabular}{|c|c|c|c|c|c|c|c|c|c|c|}
\hline \multirow[b]{2}{*}{$\begin{array}{l}\text { Type of } \\
\text { Sediment }\end{array}$} & \multicolumn{4}{|c|}{ Cr. $10^{-4}$} & \multicolumn{2}{|c|}{ Ni. $10^{-4}$} & \multicolumn{4}{|c|}{ V. $10^{-4}$} \\
\hline & & $\begin{array}{l}\text { intity } \\
\text { aples }\end{array}$ & $\begin{array}{l}\text { Limits of } \\
\text { Contents }\end{array}$ & Average & $\begin{array}{l}\text { Quantity } \\
\text { Samples }\end{array}$ & $\begin{array}{l}\text { Limits of } \\
\text { Contents }\end{array}$ & Average & $\begin{array}{l}\text { Quantity } \\
\text { Samples }\end{array}$ & $\begin{array}{l}\text { Limits of } \\
\text { Contents }\end{array}$ & Average \\
\hline $\begin{array}{l}\text { Terrigenous sediments } \\
\left(<10 \% \mathrm{CaCO}_{3}\right)\end{array}$ & $\begin{array}{l}\mathrm{a} \\
\mathrm{b}\end{array}$ & $\begin{array}{l}103 \\
103\end{array}$ & $\begin{array}{l}<20-850 \\
<20-850\end{array}$ & $\begin{array}{l}78 \\
81\end{array}$ & $\begin{array}{l}103 \\
103\end{array}$ & $\begin{array}{l}2->500 \\
2>511\end{array}$ & $\begin{array}{l}46 \\
48\end{array}$ & $\begin{array}{l}100 \\
100\end{array}$ & $\begin{array}{l}<10-400 \\
<10-494\end{array}$ & $\begin{array}{r}97 \\
102\end{array}$ \\
\hline $\begin{array}{l}\text { (and terrigenous } \\
\text { pelitic muds) }\end{array}$ & $\begin{array}{l}\mathrm{a} \\
\mathrm{b}\end{array}$ & $\begin{array}{l}22 \\
22\end{array}$ & $\begin{array}{l}43-166 \\
47-173\end{array}$ & $\begin{array}{l}101 \\
106\end{array}$ & $\begin{array}{l}22 \\
22\end{array}$ & $\begin{array}{l}2 \rightarrow 500 \\
2 \rightarrow 511\end{array}$ & $\begin{array}{l}78 \\
81\end{array}$ & $\begin{array}{l}22 \\
22\end{array}$ & $\begin{array}{l}100-400 \\
100-494\end{array}$ & $\begin{array}{l}180 \\
191\end{array}$ \\
\hline $\begin{array}{l}\text { Mixed biogenic-terrig. } \\
\left(30-50 \% \mathrm{CaCO}_{3}\right)\end{array}$ & $\begin{array}{l}\mathrm{a} \\
\mathrm{b}\end{array}$ & $\begin{array}{l}43 \\
43\end{array}$ & $\begin{array}{r}9-250 \\
18-403\end{array}$ & $\begin{array}{l}53 \\
88\end{array}$ & $\begin{array}{l}42 \\
42\end{array}$ & $\begin{array}{l}10-110 \\
16-177\end{array}$ & $\begin{array}{l}28 \\
50\end{array}$ & $\begin{array}{l}41 \\
41\end{array}$ & $\begin{array}{r}<20-160 \\
32-260\end{array}$ & $\begin{array}{r}67 \\
111\end{array}$ \\
\hline $\begin{array}{l}\text { Foram-sands } \\
\text { nanno-foram oozes } \\
\left(<50 \% \mathrm{CaCO}_{3}\right)\end{array}$ & $\begin{array}{l}\mathrm{a} \\
\mathrm{b}\end{array}$ & $\begin{array}{l}81 \\
81\end{array}$ & $\begin{array}{r}<10-250 \\
41-793\end{array}$ & $\begin{array}{r}47 \\
193\end{array}$ & $\begin{array}{l}80 \\
80\end{array}$ & $\begin{array}{l}<5-101 \\
21-381\end{array}$ & $\begin{array}{r}26 \\
109\end{array}$ & $\begin{array}{l}82 \\
81\end{array}$ & $\begin{array}{r}5-180 \\
16-866\end{array}$ & $\begin{array}{r}43 \\
182\end{array}$ \\
\hline $\begin{array}{l}\text { Diatom ooze } \\
\left(>30 \% \mathrm{SiO}_{2} \text { amorph }\right.\end{array}$ & $\begin{array}{l}\mathrm{a} \\
\mathrm{b}\end{array}$ & $\begin{array}{l}8 \\
8\end{array}$ & $\begin{array}{l}55-110 \\
56-117\end{array}$ & $\begin{array}{l}73 \\
76\end{array}$ & $\begin{array}{l}8 \\
8\end{array}$ & $\begin{array}{l}8-107 \\
9-111\end{array}$ & $\begin{array}{l}82 \\
85\end{array}$ & $\begin{array}{l}8 \\
8\end{array}$ & $\begin{array}{l}113-570 \\
118-590\end{array}$ & $\begin{array}{l}323 \\
337\end{array}$ \\
\hline $\begin{array}{l}\text { Volcanogenic } \\
\text { sediments } \\
\text { Iceland area }\end{array}$ & $\begin{array}{l}\mathrm{a} \\
\mathrm{b}\end{array}$ & $\begin{array}{l}27 \\
27\end{array}$ & $\begin{array}{l}23->1000 \\
29>1000\end{array}$ & $\begin{array}{l}145 \\
155\end{array}$ & $\begin{array}{l}26 \\
26\end{array}$ & $\begin{array}{l}<10-67 \\
<15-74\end{array}$ & $\begin{array}{l}35 \\
38\end{array}$ & $\begin{array}{l}26 \\
26\end{array}$ & $\begin{array}{r}<20-400 \\
23-420\end{array}$ & $\begin{array}{l}212 \\
228\end{array}$ \\
\hline $\begin{array}{l}\text { Red Clays } \\
\left(<10 \% \mathrm{CaCO}_{3}\right.\end{array}$ & $\begin{array}{l}\mathrm{a} \\
\mathrm{b}\end{array}$ & - & - & - & - & - & - & - & $\begin{array}{l}- \\
-\end{array}$ & $\begin{array}{l}- \\
-\end{array}$ \\
\hline $\begin{array}{l}\text { All types of } \\
\text { sediments }\end{array}$ & $\begin{array}{l}\mathrm{a} \\
\mathrm{b}\end{array}$ & $\begin{array}{l}359 \\
359\end{array}$ & $\begin{array}{l}<9-1000 \\
13->1000\end{array}$ & $\begin{array}{r}71 \\
114\end{array}$ & $\begin{array}{l}354 \\
354\end{array}$ & $\begin{array}{l}2>500 \\
2->511\end{array}$ & $\begin{array}{l}35 \\
39\end{array}$ & $\begin{array}{l}353 \\
353\end{array}$ & $\begin{array}{r}5-570 \\
10-590\end{array}$ & $\begin{array}{r}89 \\
133\end{array}$ \\
\hline
\end{tabular}

Note: $\mathrm{C}-\mathrm{C}_{\mathrm{org}}, \mathrm{Fe}, \mathrm{Mn}, \mathrm{Ti}$, and $\mathrm{P}$ for deep water muds of the preantarctic zone of the ocean (Emelyanov and others., $-\mathrm{Ba}, \mathrm{Zr}, \mathrm{Cr}, \mathrm{Ni}$,

$\mathrm{V}$, and $\mathrm{Cu}$ for shallow water muds of the southwest coast of Africa (Emelyanov, 1973)

Section 5) where limestones and marls, enriched in pyroclastics accumulated. The carbonates are characterized by a peculiar chemical composition, with properties in common with volcanoclastic sediments of basaltic composition. $\mathrm{Ti}(1.63-2.00 \%)^{\prime}$ and $\mathrm{Cr}(431-796$ $\mathrm{ppm}$ ) contents are high, as are $\mathrm{Ni}, \mathrm{Co}, \mathrm{P}, \mathrm{Mn}$, and $\mathrm{Mo}$. Like volcanoclastic sediments, they are characterized by low concentrations of $\mathrm{Fe}(3.22-6.54 \%)$.

In some cases, the chemical composition of sediment was affected by endogenic (hydrothermal) processes. Chalk deposits at the bottom of Site 355 (Core 19, Section 2 and Core 20, Section 2) overlie basalt substrate (Core 21, Section 1). The sediments, influenced by the products of hydrothermal activity, contain high quantities of $\mathrm{Fe}, \mathrm{Zn}, \mathrm{Cu}, \mathrm{Ni}$, and $\mathrm{Co}$ and low quantities of $\mathrm{Ti}, \mathrm{Na}_{2} \mathrm{O}_{3}, \mathrm{~K}_{2} \mathrm{O}$, and $\mathrm{Cr}$. Such ferruginous sediments, formed by means of endogenic sources, have been described from surface samples in the South Atlantic Ridge area (Boström et al., 1969; Boström et al., 1972), and in certain other recent sediments (Emelyanov, 1975).

Ferruginous sediments were found in the cores and dredge material recovered from the Iceland Plateau and Norwegian Sea during cruises on the R/V Academic Kurchatov. The age of these sediments ranges from early Miocene to Pleistocene. They are represented by tuffoargillites, tuffosandstones, sandstones, terrigenous (glacial) clays, and ferromanganese crusts. The following contents are characteristic of these orebearing sediments: $\mathrm{Fe}$, up to $19 \%$; $\mathrm{Mn}$, up to $17.36 \%$; $\mathrm{Ti}$, up to $0.83 \%$; $\mathrm{P}$, up to $0.35 \%$; $\mathrm{Ni}$, up to $1000 \mathrm{ppm}$; $\mathrm{Co}$, up to $500 \mathrm{ppm}$; $\mathrm{Cu}$, up to $440 \mathrm{ppm}$; V, up to 240

'Here and hereafter evaluated on carbonate-free basis. ppm; $\mathrm{Zn}$, up to $525 \mathrm{ppm} ; \mathrm{Ba}$, up to $720 \mathrm{ppm}$; Mo, up to $100 \mathrm{ppm} ; \mathrm{Cr}$, up to $74 \mathrm{ppm} ; \mathrm{Zr}$, up to $160 \mathrm{ppm} ; \mathrm{CaCO}_{3}$ is absent. As a rule, the ratio $(\mathrm{Fe}+\mathrm{Mn}) / \mathrm{Ti}$ (Strahov, 1974), a good indicator of endogenic processes for areas of the open ocean and the Mid-Atlantic Ridge, is over 25 . Although the value is not over 25 in the sediments of Site 355 (Core 19, Section 2 and Core 20, Section 2), it is close to it (ranging from Core 17, Section 21). The upper chalk deposits at Site 355 (Core 18, Section 3 up to Core 17, Section 3) were also enriched in iron and manganese by hydrothermal activity $(\mathrm{Fe}, 4.01-7.05 \%$; and $\mathrm{Mn}, 0.20-0.62 \%$ ). This enrichment is less pronounced up the section. The influence of hydrothermal action on the chemical composition of sediments in the Cretaceous is further indicated by the presence of calcite veins associated with reddish-brown ferruginous nannofossil ooze. By early Eocene hydrothermal activity had completely ceased.

A second zone enriched in ore matter as a result of volcanic activity is the layer of dolomites, occurring in conjunction with a volcanic breccia at Site 357 (Core 24, Section 6). These dolomites are overlain and underlain by limestones, are associated with pyroclastics, and contain the highest quantities of $\mathrm{Fe}$ (21.36-28.58\%) of all studied samples. They also contain high quantities of $\mathrm{Mn}, \mathrm{Zn}$, and $\mathrm{Ni}$. However, $\mathrm{Ti}$ and $\mathrm{Cr}$, which are characteristic of pyroclastics and volcanoclastic sediments of basaltic composition, occur very rarely in the dolomites. Therefore we may conclude that the dolomites were formed as the result of a combination of several factors: (1) supply of pyroclastics (found in quantity up to $5 \%$; see description of the smear slide of Sample 357-24-6, 140 $\mathrm{cm}$; (2) emanation of ore matter by means of hydrothermal action, associated with subjacent volcanic 
TABLE 8 - Continued

\begin{tabular}{ccc}
\hline & Cu.10 & \\
Quantity & Limits of & \\
Samples & Contents & Average \\
\hline 26 & $14-234$ & 75 \\
26 & $15-253$ & 80 \\
7 & $50-135$ & 86 \\
7 & $51-150$ & 90 \\
10 & $31-82$ & 54 \\
10 & $50-152$ & 93 \\
32 & $11-88$ & 31 \\
32 & $16-640$ & 178 \\
& & - \\
- & - & - \\
11 & - & 55 \\
11 & $30-99$ & 62 \\
& & \\
- & - & - \\
- & - & - \\
105 & $11-264$ & 54 \\
105 & $15-640$ & 108 \\
\hline
\end{tabular}

breccias; (3) possible chemical precipitation of dolomite and authigenic calcite from sea water. The range of the ratio $(\mathrm{Fe}+\mathrm{Mn}) / \mathrm{Ti}$, being 17 to 23 , is less than in ferruginous sediments of endogenic origin (where it usually exceeds 25 ), but greater than in pyroclastic sediments (where it usually equals 5-10, Emelyanov, 1975). This suggests that both fluid and solid volcanic source products supplied the $\mathrm{Fe}, \mathrm{Mn}$, and $\mathrm{Zn}$ and also $\mathrm{Ti}$ and $\mathrm{Co}$.

Leg 39 shipboard scientists concluded that mudstones and marly chalk of Late Cretaceous age (Maestrichtian) are also enriched in ore matter. Laboratory study shows clearly that these sediments contain neither high contents of $\mathrm{Fe}, \mathrm{Mn}, \mathrm{Zn}, \mathrm{Ni}$, or $\mathrm{Co}$ nor low contents of $\mathrm{Ti}, \mathrm{Cr}$, or $\mathrm{Al}_{2} \mathrm{O}_{3}$. $(\mathrm{Fe}+\mathrm{Mn}) / \mathrm{Ti}$ ratio is very low in these sediments (10-11), and is more typical of normal terrigenous or biogenic calcareous sediments (Emelyanov, 1975). A hydrothermal influence is evidenced by relatively high contents of $\mathrm{Fe}$ (7.10\%) and $\mathrm{Mn}(0.15 \%)$ in sediments of Cretaceous age at Site 354 (Core 17, Section 2).

Some of the chalk sediments are enriched in organic matter and contain sapropelic interlayers (Site 356, Core 41 , Section 4). In addition to the high $\mathrm{C}_{\text {org }}$ content (up to $9.8 \%$ ) they are also characterized by high values of $\mathrm{Ni}$ and $\mathrm{Zn}$ and low concentrations of $\mathrm{Mn}$. Ni, and $\mathrm{Zn}$, probably precipitated in the form of organometallic compounds. The low content of $\mathrm{Mn}$ is generally characteristic of shallow reduced muds (Emelyanov, 1973; Emelyanov et al, 1975).

The chemical composition of marly limestones of Santonian age at Site 357 (Core 48, Section 1 up to Core 51 , Section 3) is rather unusual in that the sediments contain very low concentrations of $\mathrm{Fe}, \mathrm{Zn}$, $\mathrm{Ni}$, and $\mathrm{Co}$, and low concentrations of $\mathrm{Ti}, \mathrm{Cu}, \mathrm{Cr}$, and other elements. Most likely this has been caused by admixture of considerable biogenic siliceous material, since the sediments are high in $\mathrm{SiO}_{2}$ and low in $\mathrm{Al}_{2} \mathrm{O}_{3}$ (Site 357, Core 51, Section 3).
TABLE 9

Content of Some Elements in Bottom Sediments From Leg 39

Sample

(Interval

$\begin{array}{lllllllll}\text { in } \mathrm{cm}) & \mathrm{Ba} & \mathrm{Zr} & \mathrm{V} & \mathrm{Sn} & \mathrm{Mo} & \mathrm{Be} & \mathrm{Ge}\end{array}$

\begin{tabular}{|c|c|c|c|c|c|c|}
\hline \multicolumn{7}{|l|}{ Site 354} \\
\hline $3-1,120$ & $<200$ & 80 & 70 & 6.5 & 3.7 & $<5$ \\
\hline $4-1,40-42$ & $<200$ & 60 & 80 & $<5.0$ & 3.6 & $<5$ \\
\hline $4-2,40-42$ & $<200$ & 34 & 51 & 11.0 & 2.5 & $<5$ \\
\hline $5-2,71-73$ & $<200$ & 70 & 82 & $<5.0$ & 4.0 & $<5$ \\
\hline $6-2,107$ & $<200$ & 60 & 59 & 10.0 & 3.1 & $<5$ \\
\hline $6-3,91$ & $<200$ & $<40$ & 32 & 13.0 & 2.5 & $<5$ \\
\hline $7-1,137$ & $<200$ & $<40$ & 29 & 12.0 & $<1.0$ & $<5$ \\
\hline $7-3,16$ & $<200$ & 60 & 53 & 10.0 & 2.6 & $<5$ \\
\hline $8-2,30$ & $<200$ & $<40$ & 48 & 5.7 & $<1.0$ & $<5$ \\
\hline $9-3,112$ & 840 & $<40$ & 62 & 6.4 & $<1.0$ & $<5$ \\
\hline $10-2,98$ & 830 & $<40$ & 30 & 18 & $<1.0$ & $<5$ \\
\hline $11-5,25$ & 970 & 50 & 35 & 10.0 & 2.6 & $<5$ \\
\hline $12-4,11$ & 960 & $<40$ & 37 & 7.8 & 2.5 & $<5$ \\
\hline $13-6,125$ & 960 & 50 & 31 & 8.0 & 3.2 & $<5$ \\
\hline 141,106 & 990 & $<40$ & 13 & 7.8 & 2.1 & $<5$ \\
\hline $15-2,45$ & 1300 & 60 & 19 & 8.7 & 2.5 & $<5$ \\
\hline $15-3,84$ & 980 & $<40$ & 27 & 7.9 & 2.2 & $<5$ \\
\hline $17-2,117$ & 980 & $<40$ & 37 & 6.3 & 2.3 & $<5$ \\
\hline $18-1,130$ & 1000 & $<40$ & 38 & $<5.0$ & 1.2 & $<5$ \\
\hline \multicolumn{7}{|l|}{ Site 355} \\
\hline $1-2,69-71$ & 350 & 110 & 180 & $<5.0$ & $<1.0$ & $<5$ \\
\hline $1-6,80-82$ & 350 & 130 & 150 & $<5.0$ & $<1.0$ & $<5$ \\
\hline $2-3,130-132$ & 320 & 90 & 180 & $<5.0$ & $<1.0$ & $<5$ \\
\hline $2-5,135-137$ & 220 & 70 & 140 & $<5.0$ & $<1.0$ & $<5$ \\
\hline $3-2,88-90$ & 300 & 60 & 110 & $<5.0$ & $<1.0$ & $<5$ \\
\hline $3-5,58-60$ & 300 & 100 & 130 & $<5.0$ & $<1.0$ & $<5$ \\
\hline $4-3,110-112$ & 250 & 90 & 140 & $<5.0$ & $<1.0$ & $<5$ \\
\hline $5-1,40-42$ & $<200$ & 70 & 130 & $<5.0$ & $<1.0$ & $<5$ \\
\hline $5-4,98-100$ & $<200$ & 90 & 150 & $<5.0$ & $<1.0$ & $<5$ \\
\hline $6-2,100-102$ & 300 & 70 & 110 & $<5.0$ & $<1.0$ & $<5$ \\
\hline $7-3,100-102$ & $<200$ & 60 & 170 & $<5.0$ & $<1.0$ & $<5$ \\
\hline $8-2,140-141$ & $<200$ & 130 & 120 & $<5.0$ & $<1.0$ & $<5$ \\
\hline $9-2,50-52$ & $<200$ & 200 & 110 & $<5.0$ & $<1.0$ & $<5$ \\
\hline $11-2,91-92$ & $<200$ & 130 & 100 & $<5.0$ & $<1.0$ & $<5$ \\
\hline $12-4,60-62$ & 320 & 120 & 110 & $<5.0$ & $<1.0$ & $<5$ \\
\hline $13-3,52-54$ & $<200$ & 140 & 160 & $<5.0$ & $<1.0$ & $<5$ \\
\hline $14-3,93-94$ & $<200$ & 170 & 280 & $<5.0$ & $<1.0$ & $<5$ \\
\hline $14-5,87-89$ & $<200$ & 140 & 220 & $<5.0$ & $<1.0$ & $<5$ \\
\hline $15-1,84$ & $<200$ & 250 & 870 & $<5.0$ & $<1.0$ & $<5$ \\
\hline $15-1,127$ & $<200$ & 100 & 120 & $<5.0$ & $<1.0$ & $<5$ \\
\hline
\end{tabular}

The composition of pelagic zeolithic claystones and mudstones of Eocene age (Site 355), with low contents of $\mathrm{Mn}, \mathrm{Fe}, \mathrm{P}$, and several other elements, is quite different from mid-Pleistocene muds and red clays of the Brazil Basin. It is proposed that they may not be paleo red clays, but pelagic terrigenous muds, accumulated at the depths below the compensation level.

\section{ACKNOWLEDGMENTS}

The author expresses gratitude to Dr. P. Supko, Co-chief scientist, Leg 39, for providing samples and for considerable assistance in preparation of this report and improving the English. The author also expresses gratitude to Dr. Yu. Neprochnov for performing shipboard sampling and for assistance in preparation of this work. I am very much obliged to colleagues and assistants, who conducted the chemical analyses.

\section{REFERENCES}

Bader, R.G. et al., 1970. Site 23. In Bader, R.G., Gerard, R.D., et al., Initial Reports of the Deep Sea Drilling 
TABLE 10

Content of Rare Elements in the Recent Sediments of the Atlantic Ocean near Leg 39 Sites, $\left(10^{-4} \%\right)$

\begin{tabular}{|c|c|c|c|c|c|c|c|c|c|c|}
\hline Station & $\begin{array}{l}\text { Horizon } \\
\text { (in } \mathrm{cm} \text { ) }\end{array}$ & $\mathrm{Ba}$ & $\mathrm{Cr}$ & $\mathrm{Zr}$ & $\mathrm{Ni}$ & V & Mo & $\mathrm{Ge}$ & $\mathrm{Be}$ & $\mathrm{Sn}$ \\
\hline 375 & $0-3$ & $\begin{array}{c}200 \\
(460)\end{array}$ & $\begin{array}{c}66 \\
(150)\end{array}$ & $\begin{array}{c}180 \\
(410)\end{array}$ & $\begin{array}{c}46 \\
(105)\end{array}$ & $\begin{array}{c}820 \\
(1870)\end{array}$ & $\begin{array}{l}<50 \\
(<114)\end{array}$ & $\begin{array}{l}<50 \\
(<114)\end{array}$ & $\begin{array}{l}<1.0 \\
(<2.3)\end{array}$ & $\begin{aligned}<6.0 \\
(<13.6)\end{aligned}$ \\
\hline $400-2$ & $0-7$ & $\begin{array}{l}<200 \\
(<200)\end{array}$ & $\begin{array}{c}102 \\
(103)\end{array}$ & $\begin{array}{c}90 \\
(90)\end{array}$ & $\begin{array}{c}65 \\
(66)\end{array}$ & $\begin{array}{c}1910 \\
(1920)\end{array}$ & $\begin{array}{l}<50 \\
(<50)\end{array}$ & $\begin{array}{l}<50 \\
(<50)\end{array}$ & $\begin{array}{l}<1.0 \\
(<1.0)\end{array}$ & $\begin{array}{l}<6.0 \\
(<6.0)\end{array}$ \\
\hline
\end{tabular}

Project, Volume 4: Washington (U.S. Government Printing Office), p. 17-35.

Berger, W.G., von Rad, U., 1972. Cretaceous and Cenozoic sediments from the Atlantic Ocean. In Hayes, D.E., Pimm, A.C., et al., Initial Reports of the Deep Sea Drilling Project, Volume 14: Washington (U.S. Government Printing Office), p. 787-954.

Boström, K., Peterson, M.N.A., Joensuu, O., and Fisher, D.E., 1969. Aluminium-poor ferromagnesian sediments on active oceanic ridges: J. Geophys. Res., v. 74, p. 32613270 .

Boström, K., Joensuu, O., Valdes, S., and Riera, M., 1972. Geochemical history of South Atlantic ocean sediments since Late Cretaceous: Marine Geol., v. 12, p. 85-121.

Bulatov, R.P., 1971. On the structure and circulation of the bottom layer in the Atlantic Ocean. In Condition of sedimentation of the Atlantic Ocean: Moscow (Nauka), p. 43-59.

Emelyanov, E.M., 1973. Composition of low-phosphatic and phosphatic sediments of the West African Shelf. In Formation of biologic productivity and bottom sediments in respect with peculiarities of water circulation in the southeast part of the Atlantic Ocean, IOAN Reports, v. 95, Kaliningrad (Publishing House of Kaliningrad), p. $259-260$.

, 1973. Ooze distribution and composition on the southwestern African shelf. In Formation of biologic productivity and bottom sediments in respect with peculiarities of water circulation in the southeast part of the Atlantic Ocean, IOAN Reports, v. 95, Kaliningrad (Publishing House of Kaliningrad), p. 211-238.

1975. Organic carbon in sediments of the Atlantic Ocean: AN U.S.S.R. Reports, v. 220, p. 1186-1189.

Emelyanov, E.M., Lisitzin, A.P., and Iljin, A.V., 1975. Types of bottom sediments of the Atlantic Ocean: Kaliningrad (Kaliningradskaya pravda), p. 1-570.
Ewing, M., Eittreim, S.L., Ewing, J.J., and Le Pichon, X., 1971. Sediment transport and distribution in the Argentine Basin, 3. Nepheloid layer and processes of sedimentation. In Ahrens, L.H. et al. (Eds.), Physics and Chemistry of the Earth, 8th edition: New York (Pergamon Press), p. 49.

Gietzenauer, K.R., Margolis, S.V., and Edwards, D.S., 1968. Evidence consistent with Eocene glaciation in a South Pacific deep-sea sedimentary core: Earth Planet. Sci. Lett., v. 4 , p.

Lisitzin, A.P., 1971. Distribution of carbonate microfossils in suspended matter and bottom sediments. In Funnell, B.M., and Riedel, W.R. (Eds.), Micropaleontology of Oceans: Cambridge (Cambridge University Press), p. 197 217.

Neuman, G. and Pierson, W.J., Jr., 1966. Principles of Physical Oceanography: Englewood Cliffs (Prentice-Hall, Inc.), p. 1-545.

Pimm, A.C., 1970. Carbon carbonate results, Leg 3. In Maxwell, A.E. et al., Initial Reports of the Deep Sea Drilling Project, Volume 3: Washington (U.S. Government Printing Office), p. 495-508.

Pimm, A.C., 1970. Carbon carbonate results, Leg 4. In Bader, R.G., Gerard, R.D., et al., Initial Reports of the Deep Sea Drilling Project, Volume 4: Washington (U.S. Government Printing Office), p. 307-314.

Sclater, J.G., Anderson, R.N., and Bell, M.L., 1971. Elevation of ridges and evolution of the central eastern Pacific: J. Geophys. Res., v. 76, p. 7888-7915.

Sokolov, V.S. and Sokolova, E.G., 1975. Volumetric methods of determining carbonates and carbon of organic matters in marine sediments: Moscow (Nauka), p. 19-24.

Strahov, N.M., 1974. On the exhalations at mid-oceanic ridges as the sources of ore-bearing elements in oceanic sediments: Lithology and useful minerals, v. 3, p. 20-37. 